NASA Technical Memorandum 107694

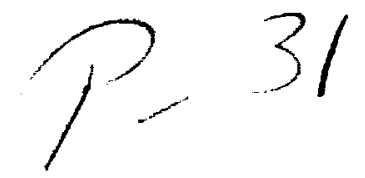

\title{
DECENTRALIZED CONTROL OF LARGE FLEXIBLE STRUCTURES BY JOINT DECOUPLING
}

\author{
NASA Technical Memorandum 107694
}

P

.

Tzu-Jeng Su and Jer-Nan Juang

October 1992

\section{N/Sก}

National Aeronautics and Space Administration

Langley Research Center Hampton, Virginia 23665

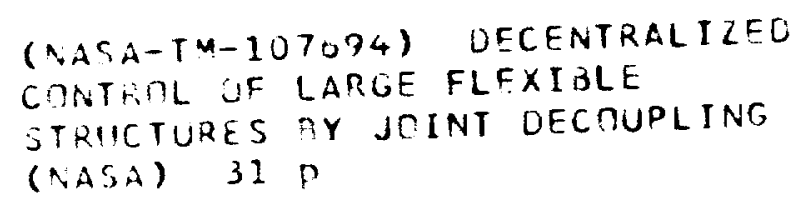

N93-12020

Unclas

$63 / 390129286$ 


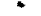




\title{
Decentralized Control Of Large Flexible Structures By Joint Decoupling
}

\author{
Tzu-Jeng Su* and Jer-Nan Juang ${ }^{\dagger}$ \\ NASA Langley Research Center \\ Hampton, Virginia 23662
}

\begin{abstract}
This paper presents a decentralized control design method for large complex flexible structures by using the idea of joint decoupling. The derivation is based on a coupled substructure state-space model, which is obtained from enforcing conditions of interface compatibility and equilibrium to the substructure state-space models. It is shown that by restricting the control law to be localized state feedback and by setting the joint actuator input commands to decouple joint degrees-of-freedom from interior degrees-of-freedom, the global structure control design problem can be decomposed into several substructure control design problems. The substructure control gains and substructure observers are designed based on modified substructure state-space models. The controllers produced by the proposed method can operate successfully at the individual substructure level as well as at the global structure level. Therefore, not only control design but also control implementation is decentralized. Stability and performance requirement of the closedloop system can be achieved by using any existing state feedback control design method. A two-component mass-spring-damper system and a three-truss structure are used as examples to demonstrate the proposed method.
\end{abstract}

\section{Introduction}

Control of flexible structures has gained much research interest since the space shuttle transportation system became reality and construction of large structures in space is no longer a dream. Many structural control methods and algorithms have been proposed in the past decade. However, the application of decentralized control to flexible structures

* National Research Council Research Associate, Member AIAA.

†Principal Scientist, Spacecraft Dynamics Branch, Fellow AIAA. 
has not been pursued extensively. A large space structure must be built incrementally with components shipped into space sequentially during several shuttle missions. Also, for operational purposes, space structures may need to be connected and disconnected routinely in space. Therefore, it is desirable to have controllers that can function well on each individual structure and also on the interconnected structures. Decentralized controllers designed based on structure components will fit to meet such purpose.

Most of existing decentralized control designed methods were developed for application to electrical engineering systems or economics systems, rather than to flexible structural dynamics systems (see, for example, Refs. [1-3]). In general, structural dynamics systems are strongly coupled (in physical coordinates), which makes it difficult to design and to implement decentralized control. Existing decentralized control methods for flexible structures either adopt or extend the concepts and methodologies developed for other type of systems. For instance, the method in Ref. [4] extends the concept of decentralized fixed modes of Ref. [1] to structural control problem. The decentralization of the control problem is based on modes instead of physical components of the structure. The controlled component synthesis (CCS) method of Ref. [5] adopts the concept of overlapping decomposition in Ref. [3] to design controllers for physical structural components. Because overlapping decomposition requires information about adjacent components, the CCS method is not a strict decentralized approach. The substructure controller synthesis (SCS) method in Ref. [6] is a decentralized control design method which uses natural decomposition of structural dynamics systems. Both CCS and SCS methods employ interface compatibility conditions to assemble the substructure controllers into a global controller for the assembled structure. However, because interface equilibrium conditions are not considered in the assembly process, the global controller does not guarantee stability of the closed-loop system, which is a major disadvantage of both CCS and SCS methods.

This paper presents a decentralized control design method based on the idea of joint decoupling. The method is developed specifically for decentralized control of large complex flexible structures. The structure to be controlled is first decomposed into several substructures, which can be physical components that are to be connected together to form the assembled structure, or they can simply be the result of applying "imaginary" cuts to the structure. It is assumed that there are collocated actuators and sensors at every interface (or joint) degree-of-freedom. Then, by enforcing conditions of compatibility and equilibrium to the joint inputs and joint outputs, state-space models of substructures are combined together to form a coupled substructure state space model. 
Then, based on the coupled substructure state-space model it is shown that control design can be decentralized by restricting the control gain matrix and the observer gain matrix to have a specific form. The whole design concept can be described as follows. The control commands for the interior actuators (i.e., actuators that are not located at the joints) of each substructure are chosen to be localized state feedback. Then, the joint actuator commands are set to cancel out all the forces acting on the joint degreesof-freedom which are induced by interior actuator inputs and by vibration of interior degrees-of-freedom. The result is that the joint degrees-of-freedom are decoupled from the interior degrees-of-freedom and, therefore, there is no interaction force transmitted through the joints from one substructure to another substructure when the substructures are connected together. The controller designed by using the idea of joint decoupling is called the Joint Decoupling Controller.

The proposed Joint Decoupling Controller has several advantages. First, the design procedure is completely decentralized. It requires no information about the other substructures in order to do control design for one substructure. Second, the control implementation is also decentralized. The controllers can operate successfully at the individual substructure level before assembly and also at the global structure level after assembly. This feature makes the proposed method extremely useful for active control of space structures that need to be connected and disconnected on a routine basis for operational purposes. No controller redesign or controller shut-off-and-turn-on is necessary before, during, or after the connection process. Third, the proposed method divides a large-scale control problem into several small-scale subproblems, and, hence, computationally it is more efficient than a centralized control design approach. The control law can be determined by using any state feedback control design method, e.g., the LQR method or pole placement method.

This paper is organized as follows. The coupled substructure state-space model is derived in Section II. The design procedure for a decentralized control gain matrix and the design procedure for a decentralized observer system are presented in Sections III and IV, respectively. Also included in Section IV is a summary of design steps for the proposed Joint Decoupling Controller. A six degrees-of-freedom mass-spring-damper system and a three-truss structure are used in Section $V$ as examples to demonstrate the proposed method. 


\section{The Coupled Substructure State-Space Model}

In this section, we will derive the coupled substructure state-space model for flexible structures that are composed of many substructures. Without loss of generality, we will consider the two-component structure shown in Fig. 1. Figure 1(a) shows the assembled

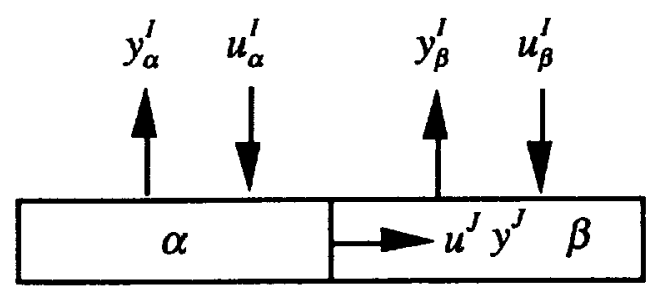

(a)
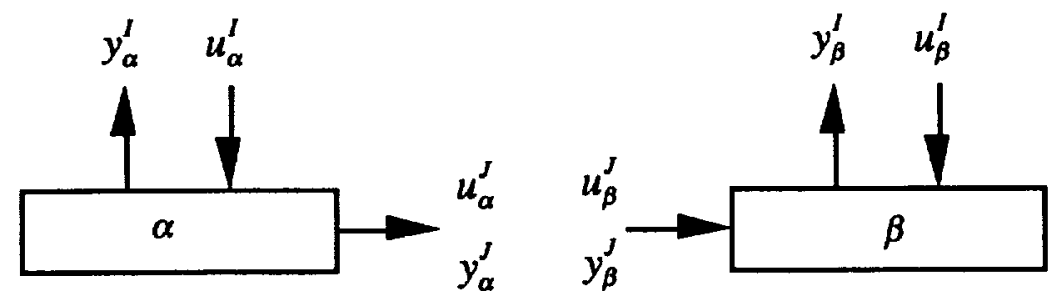

(b)

Figure 1: A two-component structure.

structure as an entity, which also will be referred to as the global structure. Figure 1(b) shows the decoupled two substructures $\alpha$ and $\beta$. These two substructures can be physical components that are to be connected together to form the structure, or they can be the result of applying an "imaginary cut" to the structure. Locations of actuators and sensors are denoted by $u$ and $y$, which are divided into two groups. Those actuators and sensors located at the joint (or interface, where the substructures are to be connected together) are denoted with a superscript $J$. Those actuators and sensors located at unconnected points (or located at "interior" points of each substructure) are denoted with a superscript I. In order to describe interface compatibility and equilibrium conditions in terms of input and output vectors, it is assumed that there are a pair of collocated actuator and sensor at every joint degree-of-freedom. Also, the output measurements at the joint coordinates are assumed to be accelerations for a special purpose to be discussed later. 
Let the state-space models of $\alpha$-substructure and $\beta$-substructure be described, respectively, by

and

$$
\begin{gathered}
\dot{x}_{\alpha}=A_{\alpha} x_{\alpha}+\left[\begin{array}{ll}
B_{\alpha}^{I} & B_{\alpha}^{J}
\end{array}\right]\left\{\begin{array}{c}
u_{\alpha}^{I} \\
u_{\alpha}^{J}
\end{array}\right\} \\
\left\{\begin{array}{c}
y_{\alpha}^{I} \\
y_{\alpha}^{J}
\end{array}\right\}=\left[\begin{array}{c}
C_{\alpha}^{I} \\
C_{\alpha}^{J}
\end{array}\right] x_{\alpha}+\left[\begin{array}{cc}
D_{\alpha}^{I I} & D_{\alpha}^{I J} \\
D_{\alpha}^{J I} & D_{\alpha}^{J J}
\end{array}\right]\left\{\begin{array}{c}
u_{\alpha}^{I} \\
u_{\alpha}^{J}
\end{array}\right\}
\end{gathered}
$$

$$
\begin{gathered}
\dot{x}_{\beta}=A_{\beta} x_{\beta}+\left[\begin{array}{ll}
B_{\beta}^{I} & B_{\beta}^{J}
\end{array}\right]\left\{\begin{array}{c}
u_{\beta}^{I} \\
u_{\beta}^{J}
\end{array}\right\} \\
\left\{\begin{array}{c}
y_{\beta}^{I} \\
y_{\beta}^{J}
\end{array}\right\}=\left[\begin{array}{c}
C_{\beta}^{I} \\
C_{\beta}^{J}
\end{array}\right] x_{\beta}+\left[\begin{array}{cc}
D_{\beta}^{I I} & D_{\beta}^{I J} \\
D_{\beta}^{J I} & D_{\beta}^{J J}
\end{array}\right]\left\{\begin{array}{c}
u_{\beta}^{I} \\
u_{\beta}^{J}
\end{array}\right\}
\end{gathered}
$$

These two state-space models can be derived from finite-element modeling of the two substructures. For instance, let the finite-element model of $\alpha$-substructure be described by

$$
\begin{aligned}
& M_{\alpha}\left\{\begin{array}{c}
\ddot{w}_{\alpha}^{I} \\
\ddot{w}_{\alpha}^{J}
\end{array}\right\}+Z_{\alpha}\left\{\begin{array}{c}
\dot{w}_{\alpha}^{I} \\
\dot{w}_{\alpha}^{J}
\end{array}\right\}+K_{\alpha}\left\{\begin{array}{c}
w_{\alpha}^{I} \\
w_{\alpha}^{J}
\end{array}\right\}=\left[\begin{array}{cc}
P_{\alpha}^{I} & 0 \\
0 & I
\end{array}\right]\left\{\begin{array}{c}
u_{\alpha}^{I} \\
u_{\alpha}^{J}
\end{array}\right\} \\
& \left\{\begin{array}{c}
y_{\alpha}^{I} \\
y_{\alpha}^{J}
\end{array}\right\}=\left[\begin{array}{cc}
H_{\alpha}^{I} & 0 \\
0 & I
\end{array}\right]\left\{\begin{array}{c}
\ddot{w}_{\alpha}^{I} \\
\ddot{w}_{\alpha}^{J}
\end{array}\right\}
\end{aligned}
$$

where $w_{\alpha}^{I}$ and $w_{\alpha}^{J}$ are the interior and the joint displacement coordinates, respectively. $M_{\alpha}, Z_{\alpha}$, and $K_{\alpha}$ are the substructure mass, damping, and stiffness matrices. $P_{\alpha}^{I}$ is the interior actuator distribution matrices. $H_{\alpha}^{I}$ is the interior accelerometer distribution matrices. (For more general cases, the interior outputs may also include displacements and velocities.) The identity matrix $I$ in the input and output matrices indicates that there are actuators and sensors collocated at every joint degrees-of-freedom. In first-order state-space form, the system in Eq. (3) can be represented by

$$
\begin{aligned}
& \dot{x}_{\alpha}=\left[\begin{array}{cc}
0 & I \\
-M_{\alpha}^{-1} K_{\alpha} & -M_{\alpha}^{-1} Z_{\alpha}
\end{array}\right] x_{\alpha}+\left[\begin{array}{cc}
0 & 0 \\
M_{\alpha}^{-1}\left[\begin{array}{c}
P_{\alpha}^{I} \\
0
\end{array}\right] & M_{\alpha}^{-1}\left[\begin{array}{l}
0 \\
I
\end{array}\right]
\end{array}\right]\left\{\begin{array}{c}
u_{\alpha}^{I} \\
u_{\alpha}^{J}
\end{array}\right\} \\
& \left\{\begin{array}{l}
y_{\alpha}^{I} \\
y_{\alpha}^{J}
\end{array}\right\}=\left[\begin{array}{cc}
H_{\alpha}^{I} & 0 \\
0 & I
\end{array}\right]\left[-M_{\alpha}^{-1} K_{\alpha}-M_{\alpha}^{-1} Z_{\alpha}\right] x_{\alpha}+\left[\begin{array}{cc}
H_{\alpha}^{I} & 0 \\
0 & I
\end{array}\right] M_{\alpha}^{-1}\left[\begin{array}{cc}
P_{\alpha}^{I} & 0 \\
0 & I
\end{array}\right]\left\{\begin{array}{l}
u_{\alpha}^{I} \\
u_{\alpha}^{J}
\end{array}\right\}
\end{aligned}
$$

where the state vector is defined by

$$
x_{\alpha}=\left\{\begin{array}{c}
w_{\alpha}^{I} \\
w_{\alpha}^{J} \\
\dot{w}_{\alpha}^{I} \\
\dot{w}_{\alpha}^{J}
\end{array}\right\}
$$


For convenience of derivation in this paper, the substructure state vector is rearranged as

$$
x_{\alpha}=\left\{\begin{array}{c}
w_{\alpha}^{I} \\
\dot{w}_{\alpha}^{I} \\
w_{\alpha}^{J} \\
\dot{w}_{\alpha}^{J}
\end{array}\right\} \equiv\left\{\begin{array}{c}
x_{\alpha}^{I} \\
x_{\alpha}^{J}
\end{array}\right\}
$$

and the system matrices are rearranged accordingly. Other than finite-element modeling, the substructure state-space models also can be realized directly from substructure experimental data by using system identification tools.

The two state-space models in Eqs. (1) and (2) describe the dynamics of the two substructures when they are completely decoupled. When the two substructures are joined together to form the global structure, the input and output vectors at the interface of the two substructures are no longer independent. They must satisfy two conditions: compatibility and equilibrium. The output vectors at the interface are constrained by the compatibility equation:

$$
y_{\alpha}^{J}=y_{\beta}^{J}=y^{J}
$$

which says that the physical motion of the two substructure at the interface must be the same. The input vectors at the interface are related by the equilibrium equation:

$$
u^{J}=u_{\alpha}^{J}+u_{\beta}^{J}
$$

which says that the sum of the internal forces at the interface must be equal to the external force applied there. The two substructure state-space models can be coupled by enforcing interface compatibility and equilibrium conditions. First, rewrite the bottom part of the output equations in Eqs. (1) and (2) as

$$
\begin{aligned}
& u_{\alpha}^{J}=\left(D_{\alpha}^{J J}\right)^{-1}\left(y_{\alpha}^{J}-C_{\alpha}^{J} x_{\alpha}-D_{\alpha}^{J I} u_{\alpha}^{I}\right) \\
& u_{\beta}^{J}=\left(D_{\beta}^{J J}\right)^{-1}\left(y_{\beta}^{J}-C_{\beta}^{J} x_{\beta}-D_{\beta}^{J I} u_{\beta}^{I}\right)
\end{aligned}
$$

Adding the above two equations and applying the equilibrium and compatibility conditions in Eq. (7), we obtain

$$
u^{J}=\left[\left(D_{\alpha}^{J J}\right)^{-1}+\left(D_{\beta}^{J J}\right)^{-1}\right] y^{J}-\left(D_{\alpha}^{J J}\right)^{-1}\left(C_{\alpha}^{J} x_{\alpha}+D_{\alpha}^{J I} u_{\alpha}^{I}\right)-\left(D_{\beta}^{J J}\right)^{-1}\left(C_{\beta}^{J} x_{\beta}+D_{\beta}^{J I} u_{\beta}^{I}\right)
$$

The above equation can be rewritten as

$$
y^{J}=D_{\alpha}^{J J} S^{-1} D_{\beta}^{J J}\left[u_{\alpha}^{J}+u_{\beta}^{J}+\left(D_{\alpha}^{J J}\right)^{-1}\left(C_{\alpha}^{J} x_{\alpha}+D_{\alpha}^{J I} u_{\alpha}^{I}\right)+\left(D_{\beta}^{J J}\right)^{-1}\left(C_{\beta}^{J} x_{\beta}+D_{\beta}^{J I} u_{\beta}^{I}\right)\right]
$$


where $S=\left(D_{\alpha}^{J J}+D_{\beta}^{J J}\right)$ and $u^{J}$ is replaced by $u_{\alpha}^{J}+u_{\beta}^{J}$. Finally, by substituting Eq. (9) into Eqs. (8) and then substituting the results into the two substructure state-space models in Eqs. (1) and (2), we obtain the following "combined" state-space model for the two substructures:

$$
\begin{aligned}
& \left\{\begin{array}{c}
\dot{x}_{\alpha} \\
\dot{x}_{\beta}
\end{array}\right\}=\left[\begin{array}{cc}
A_{\alpha}-B_{\alpha}^{J} S^{-1} C_{\alpha}^{J} & B_{\alpha}^{J} S^{-1} C_{\beta}^{J} \\
B_{\beta}^{J} S^{-1} C_{\alpha}^{J} & A_{\beta}-B_{\beta}^{J} S^{-1} C_{\beta}^{J}
\end{array}\right]\left\{\begin{array}{c}
x_{\alpha} \\
x_{\beta}
\end{array}\right\}
\end{aligned}
$$

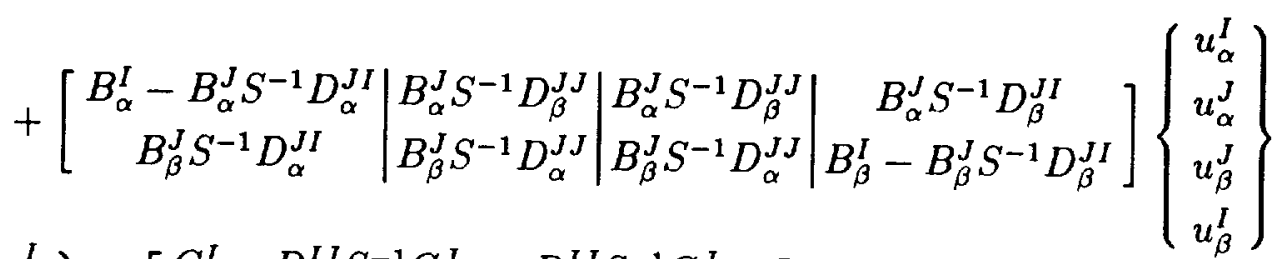

$$
\begin{aligned}
& \left\{\begin{array}{c}
y_{\alpha}^{I} \\
y_{\alpha}^{J} \\
y_{\beta}^{J} \\
y_{\beta}^{I}
\end{array}\right\}=\left[\begin{array}{cc}
C_{\alpha}^{I}-D_{\alpha}^{I J} S^{-1} C_{\alpha}^{J} & D_{\alpha}^{I J} S^{-1} C_{\beta}^{J} \\
D_{\beta}^{J J} S^{-1} C_{\alpha}^{J} & D_{\alpha}^{J J} S^{-1} C_{\beta}^{J} \\
D_{\beta}^{J J} S^{-1} C_{\alpha}^{J} & D_{\alpha}^{J J} S^{-1} C_{\beta}^{J} \\
D_{\beta}^{I J} S^{-1} C_{\alpha}^{J} & C_{\beta}^{I}-D_{\beta}^{I J} S^{-1} C_{\beta}^{J}
\end{array}\right]\left\{\begin{array}{c}
x_{\alpha} \\
x_{\beta}
\end{array}\right\} \\
& +\left[\begin{array}{c|c|c|c}
D_{\alpha}^{I I}-D_{\alpha}^{I J} S^{-1} D_{\alpha}^{J I} & D_{\alpha}^{I J} S^{-1} D_{\beta}^{J J} & D_{\alpha}^{I J} S^{-1} D_{\beta}^{J J} & D_{\alpha}^{I J} S^{-1} D_{\beta}^{J I} \\
D_{\beta}^{J J} S^{-1} D_{\alpha}^{J I} & D_{\alpha}^{J J} S^{-1} D_{\beta}^{J J} & D_{\alpha}^{J J} S^{-1} D_{\beta}^{J J} & D_{\alpha}^{J J} S^{-1} D_{\beta}^{J I} \\
D_{\beta}^{J J} S^{-1} D_{\alpha}^{J I} & D_{\alpha}^{J J} S^{-1} D_{\beta}^{J J} & D_{\alpha}^{J J} S^{-1} D_{\beta}^{J J} & D_{\alpha}^{J J} S^{-1} D_{\beta}^{J I} \\
D_{\beta}^{I J} S^{-1} D_{\alpha}^{J I} & D_{\beta}^{I J} S^{-1} D_{\alpha}^{J J} & D_{\beta}^{I J} S^{-1} D_{\alpha}^{J J} & D_{\beta}^{I I}-D_{\beta}^{I J} S^{-1} D_{\beta}^{J I}
\end{array}\right]\left\{\begin{array}{c}
u_{\alpha}^{I} \\
u_{\alpha}^{J} \\
u_{\beta}^{J} \\
u_{\beta}^{I}
\end{array}\right\}
\end{aligned}
$$

In short notation, Eq. (10) will be represented by

$$
\begin{aligned}
& \dot{\tilde{x}}=\tilde{A} \tilde{x}+\tilde{B} \tilde{u} \\
& \tilde{y}=\tilde{C} \tilde{x}+\tilde{D} \tilde{u}
\end{aligned}
$$

where $\tilde{A}, \tilde{B}, \tilde{C}, \tilde{D}, \tilde{x}, \tilde{u}$, and $\tilde{y}$ are clearly defined.

The state-space model in Eq. (10) will be referred to as the coupled substructure state-space model. It describes the dynamics of the two substructures in Fig. 1(b) with compatibility and equilibrium conditions satisfied at the interface. Therefore, it can be considered as a state-space representation of the global structure in Fig. 1(a). In fact, there is a relationship between the coupled substructure state-space model and the statespace model of the global structure. Let the state-space model of the global structure in Fig. 1(a) be described by

$$
\begin{aligned}
& \dot{x}=A x+B u \\
& y=C x+D u
\end{aligned}
$$


with the state, input, and output vectors defined by

$$
x=\left\{\begin{array}{c}
x_{\alpha}^{I} \\
x^{J} \\
x_{\beta}^{I}
\end{array}\right\} \quad, \quad u=\left\{\begin{array}{c}
u_{\alpha}^{I} \\
u^{J} \\
u_{\beta}^{I}
\end{array}\right\} \quad, \quad y=\left\{\begin{array}{c}
y_{\alpha}^{I} \\
y^{J} \\
y_{\beta}^{I}
\end{array}\right\}
$$

We see that $\tilde{x}$ and $x$ are related by a linear transformation

$$
\tilde{x} \equiv\left\{\begin{array}{c}
x_{\alpha}^{I} \\
x_{\alpha}^{J} \\
x_{\beta}^{I} \\
x_{\beta}^{J}
\end{array}\right\}=\left[\begin{array}{lll}
I & 0 & 0 \\
0 & I & 0 \\
0 & 0 & I \\
0 & I & 0
\end{array}\right]\left\{\begin{array}{l}
x_{\alpha}^{I} \\
x^{J} \\
x_{\beta}^{I}
\end{array}\right\} \quad \text { or } \quad \tilde{x}=T x
$$

where $T$ will be referred to as the state coupling matrix. Also, $\tilde{u}$ and $u$ are related by

$$
\left\{\begin{array}{c}
u_{\alpha}^{I} \\
u^{J} \\
u_{\beta}^{I}
\end{array}\right\}=\left[\begin{array}{llll}
I & 0 & 0 & 0 \\
0 & I & I & 0 \\
0 & 0 & 0 & I
\end{array}\right]\left\{\begin{array}{c}
u_{\alpha}^{I} \\
u_{\alpha}^{J} \\
u_{\beta}^{J} \\
u_{\beta}^{I}
\end{array}\right\} \quad \text { or } \quad u=T_{1} \tilde{u}
$$

$\tilde{y}$ and $y$ are related by

$$
\left\{\begin{array}{c}
y_{\alpha}^{I} \\
y_{\alpha}^{J} \\
y_{\beta}^{J} \\
y_{\beta}^{I}
\end{array}\right\}=\left[\begin{array}{lll}
I & 0 & 0 \\
0 & I & 0 \\
0 & I & 0 \\
0 & 0 & I
\end{array}\right]\left\{\begin{array}{c}
y_{\alpha}^{I} \\
y^{J} \\
y_{\beta}^{I}
\end{array}\right\} \quad \text { or } \quad \tilde{y}=T_{2} y
$$

$T_{1}$ and $T_{2}$ will be referred to as the input coupling matrix and the output coupling matrix, respectively. It should be noted that in general $T_{1} \neq T_{2}^{T}$, although it appears to be, because the number of interior sensors and the number of interior actuators can be different. By using the state, input, and output coupling matrices, it can be shown that the global structure state-space model and the coupled substructure state-space model are related by

$$
T A=\tilde{A} T \quad, \quad T B T_{1}=\tilde{B} \quad, \quad T_{2} C=\tilde{C} T \quad, \quad T_{2} D T_{1}=\tilde{D}
$$

In the preceding derivation, we have assumed that both $D_{\alpha}^{J J}$ and $D_{\beta}^{J J}$ are invertible. This is true when the outputs at the interface are accelerations. As can be seen from Eqs. (3) and (4), when the interface output is $y_{\alpha}^{J}=\ddot{w}_{\alpha}^{J}$ we get

$$
D_{\alpha}^{J J}=\left[\begin{array}{ll}
0 & I
\end{array}\right] M_{\alpha}^{-1}\left[\begin{array}{l}
0 \\
I
\end{array}\right]
$$


which is invertible because $M_{\alpha}$ is positive definite. (For most structures, the mass matrix is positive definite.) Since accelerometers are the most frequently used sensors in practical situations, this assumption is not a serious restriction. Even when the sensing devices used at the interface are displacement sensors or velocity sensors instead of accelerometers, it is still possible to perform coupling of structure state-space models by making modifications to the substructure state-space models.

In the following sections, a method for designing decentralized controllers for the global structure will be derived. It will be shown that the controller gains for joint inputs and the observer gains for joint outputs can be chosen such that the coupling between substructures is eliminated. By doing so, the global structure control problem is decomposed into two substructure control problems.

\section{The Design of Decentralized Controller Gain Matrix}

According to the separation principle, controller gain matrix and observer gain matrix can be designed separately. In this section, we will derive the procedure for designing decentralized (or localized) state feedback gain matrix for the global structure. As discussed previously, the coupled substructure state-space model in Eq. (10) is a state-space representation of the global structure. Therefore, the whole derivation will be based on the coupled substructure state-space model.

The key idea behind the decentralization method presented here is to use the joint actuators to eliminate the interaction between substructures. Let the joint actuator commands be chosen as

$$
\begin{aligned}
& u_{\alpha}^{J}=-\left(D_{\alpha}^{J J}\right)^{-1}\left(C_{\alpha}^{J} x_{\alpha}+D_{\alpha}^{J I} u_{\alpha}^{I}\right) \\
& u_{\beta}^{J}=-\left(D_{\beta}^{J J}\right)^{-1}\left(C_{\beta}^{J} x_{\beta}+D_{\beta}^{J I} u_{\beta}^{I}\right)
\end{aligned}
$$

After algebraic manipulations, substitution of the above into the state equation in Eq. (10) yields

where

$$
\left\{\begin{array}{c}
\dot{x}_{\alpha} \\
\dot{x}_{\beta}
\end{array}\right\}=\left[\begin{array}{cc}
A_{\alpha m} & 0 \\
0 & A_{\beta m}
\end{array}\right]\left\{\begin{array}{l}
x_{\alpha} \\
x_{\beta}
\end{array}\right\}+\left[\begin{array}{cc}
B_{\alpha m}^{I} & 0 \\
0 & B_{\beta m}^{I}
\end{array}\right]\left\{\begin{array}{c}
u_{\alpha}^{I} \\
u_{\beta}^{I}
\end{array}\right\}
$$

$$
\begin{array}{ll}
A_{\alpha m}=A_{\alpha}-B_{\alpha}^{J}\left(D_{\alpha}^{J J}\right)^{-1} C_{\alpha}^{J} & ,
\end{array}
$$


Subscript $m$ is used to denote that the above matrices are modified substructure matrices. Note that the system in Eq. (19) is decoupled. This decoupling of the closed-loop state equation suggests a decentralized control design procedure, which can be described as follows. First, restrict the feedback commands for the interior actuators to be localized state feedback as described by

$$
u_{\alpha}^{I}=G_{\alpha}^{I} x_{\alpha} \quad, \quad u_{\beta}^{I}=G_{\beta}^{I} x_{\beta}
$$

The gain matrices $G_{\alpha}^{I}$ and $G_{\beta}^{I}$ are to be designed based on the following modified substructure state-space models:

$$
\begin{aligned}
& \dot{x}_{\alpha}=A_{\alpha m} x_{\alpha}+B_{\alpha m}^{I} u_{\alpha}^{I} \\
& \dot{x}_{\beta}=A_{\beta m} x_{\beta}+B_{\beta m}^{I} u_{\beta}^{I}
\end{aligned}
$$

Then, according to Eq. (18), set the feedback commands for joint actuators to be

$$
\begin{aligned}
& u_{\alpha}^{J}=-\left(D_{\alpha}^{J J}\right)^{-1}\left(C_{\alpha}^{J}+D_{\alpha}^{J I} G_{\alpha}^{I}\right) x_{\alpha} \equiv G_{\alpha}^{J} x_{\alpha} \\
& u_{\beta}^{J}=-\left(D_{\beta}^{J J}\right)^{-1}\left(C_{\beta}^{J}+D_{\beta}^{J I} G_{\beta}^{I}\right) x_{\beta} \equiv G_{\beta}^{J} x_{\beta}
\end{aligned}
$$

Finally, substitute this set of actuator commands in Eqs. (21) and (23) into Eq. (15) and use the input coupling matrix to obtain a global feedback gain matrix for control implementation on the global structure.

For the decentralized control design approach described above to be successful, both modified substructure systems $\left(A_{\alpha m}, B_{\alpha m}^{I}\right)$ and $\left(A_{\beta m}, B_{\beta m}^{I}\right)$ must be controllable. Or, at least, all the unstable poles of $A_{\alpha m}$ and $A_{\beta m}$ need to be controllable so that the modified substructure systems are stabilizable. However, this is not true. Substitution of the joint actuator commands in Eq. (18) into Eq. (8) gives $y_{\alpha}^{J} \equiv \ddot{w}_{\alpha}^{J}=0$ and $y_{\beta}^{J} \equiv \ddot{w}_{\beta}^{J}=0$, which means that all the joint degrees-of-freedom are free of forces. Physically, the joint actuator commands in Eq. (18) cancel out all the internal forces acting on the joint degrees-of-freedom. The internal forces acting on the joint degrees-of-freedom are induced by interior inputs and vibration of the substructures. Cancellation of internal forces decouples the dynamics of the joint degrees-of-freedom from that of the interior degreesof-freedom. By doing so, there will be no interaction between substructures when the two substructures are connected. The only problem is that the joint degrees-of-freedom, even though free of forces, still can have rigid body motion. This rigid-body motions of the joint-degrees-of-freedom are not controllable by interior actuators, since all control forces coming from interior actuators to the joint degrees-of-freedom will be cancelled by the joint actuator commands in Eq. (18). As a result, the substructure closed-loop 
systems and the global structure closed-loop system attain a pair of unstable double poles at the origin for every joint degree-of-freedom. A simple approach to control the rigid body motions of joint degrees-of-freedom is to introduce an augmented joint actuator command. Details about the design of interior actuator feedback gain matrices, $G_{\alpha}^{I}$ and $G_{\beta}^{I}$, and the augmented joint actuator command are discussed in the following.

In physical coordinates, the modified state equation for $\alpha$-substructure can be partitioned as

$$
\left\{\begin{array}{c}
\dot{x}_{\alpha}^{I} \\
\dot{x}_{\alpha}^{J}
\end{array}\right\}=\left[\begin{array}{cc}
A_{\alpha m}^{I I} & A_{\alpha m}^{I J} \\
A_{\alpha m}^{J I} & A_{\alpha m}^{J J}
\end{array}\right]\left\{\begin{array}{c}
x_{\alpha}^{I} \\
x_{\alpha}^{J}
\end{array}\right\}+\left[\begin{array}{c}
B_{\alpha m}^{I I} \\
B_{\alpha m}^{J I}
\end{array}\right] u_{\alpha}^{I}
$$

in which the state vector $x_{\alpha}$ is partitioned according to $\mathrm{Eq}$. (5). As discussed previously, the uncontrollable state in Eq. (24) is $x_{\alpha}^{J}$. Therefore, $\left(A_{\alpha m}^{I I}, B_{\alpha m}^{I I}\right)$ is a completely controllable system, presuming that the given interior actuator setting makes the substructure controllable. We can set

$$
u_{\alpha}^{I}=G_{\alpha}^{I} x_{\alpha}=\left[\begin{array}{ll}
G_{\alpha}^{I I} & 0
\end{array}\right]\left\{\begin{array}{l}
x_{\alpha}^{I} \\
x_{\alpha}^{J}
\end{array}\right\}
$$

with $G_{\alpha}^{I I}$ to be designed based on the subsystem $\dot{x}_{\alpha}^{I}=A_{\alpha m}^{I I} x_{\alpha}^{I}+B_{\alpha m}^{I I} u_{\alpha}^{I}$. Any existing state feedback design method, e.g., the LQR control theory or pole placement method, can be used to determine $G_{\alpha}^{I I}$. Similarly, we can set $G_{\beta}^{I}=\left[G_{\beta}^{I I} 0\right]$ and let $G_{\beta}^{I I}$ be designed based on $\dot{x}_{\beta}^{I}=A_{\beta m}^{I I} x_{\beta}^{I}+B_{\beta m}^{I I} u_{\beta}^{I}$. By doing so, the controllable poles of $A_{\alpha m}$ and $A_{\beta m}$ are stabilized or controlled by $u_{\alpha}^{I}$ and $u_{\beta}^{I}$. If the substructure state-space models are identified from experimental data rather than derived from finite-element models, the system equations are in general not in physical coordinates. Then, there is no way to tell which part of the system matrices are associated with the interior degrees-of-freedom or with the joint degrees-of-freedom. In this case, it is necessary to use existing system realization algorithms to separate the system equations into controllable subsystems and uncontrollable subsystems. Then, $G_{\alpha}^{I I}$ and $G_{\beta}^{I I}$ can be designed based on the controllable subsystems.

To control the rigid-body motions of the joint degrees-of-freedom, an allgmented joint actuator command is introduced. Let

$$
\begin{aligned}
& u_{\alpha}^{J}=G_{\alpha}^{J} x_{\alpha}+\bar{u}_{\alpha}^{J} \\
& u_{\beta}^{J}=G_{\beta}^{J} x_{\beta}+\bar{u}_{\beta}^{J}
\end{aligned}
$$

where $\bar{u}_{\alpha}^{J}$ and $\bar{u}_{\beta}^{J}$ are the augmented joint inputs. Substitution of the above into Eq. (8) yields

$$
\bar{u}_{\alpha}^{J}=\left(D_{\alpha}^{J J}\right)^{-1} y_{\alpha}^{J} \equiv\left(D_{\alpha}^{J J}\right)^{-1} \ddot{w}_{\alpha}^{J}
$$




$$
\bar{u}_{\beta}^{J}=\left(D_{\beta}^{J J}\right)^{-1} y_{\beta}^{J} \equiv\left(D_{\beta}^{J J}\right)^{-1} \ddot{w}_{\beta}^{J}
$$

We can set the augmented joint inputs to be

$$
\begin{aligned}
& \bar{u}_{\alpha}^{J}=\left(D_{\alpha}^{J J}\right)^{-1}\left[-\left[\Omega_{i}^{2}\right]-\left[2 \zeta_{i} \Omega_{i}\right]\right]\left\{\begin{array}{c}
w_{\alpha}^{J} \\
\dot{w}_{\alpha}^{J}
\end{array}\right\} \equiv \bar{G}_{\alpha}^{J J} x_{\alpha}^{J} \\
& \bar{u}_{\beta}^{J}=\left(D_{\beta}^{J J}\right)^{-1}\left[-\left[\Omega_{i}^{2}\right]-\left[2 \zeta_{i} \Omega_{i}\right]\right]\left\{\begin{array}{c}
w_{\beta}^{J} \\
\dot{w}_{\beta}^{J}
\end{array}\right\} \equiv \bar{G}_{\beta}^{J J} x_{\beta}^{J}
\end{aligned}
$$

where $\left[2 \zeta_{i} \Omega_{i}\right]$ and $\left[\Omega_{i}^{2}\right]$ are diagonal matrices with the design parameters $\zeta_{i}$ and $\Omega_{i}$, $i=1,2, \ldots, n_{c}$, on the diagonal. Then, the closed-loop equations for joint degrees-offreedom become

$$
\begin{aligned}
& \ddot{w}_{\alpha}^{J}+\left[2 \zeta_{i} \Omega_{i}\right] \dot{w}_{\alpha}^{J}+\left[\Omega_{i}^{2}\right] w_{\alpha}^{J}=0 \\
& \ddot{w}_{\beta}^{J}+\left[2 \zeta_{i} \Omega_{i}\right] \dot{w}_{\beta}^{J}+\left[\Omega_{i}^{2}\right] w_{\beta}^{J}=0
\end{aligned}
$$

which show that the closed-loop poles associated with joint degrees-of-freedom are stable poles located at $-\zeta_{i} \Omega_{i} \pm-j \Omega_{i} \sqrt{1-\zeta^{2}}, i=1,2, \ldots, n_{c}$. The augmented joint actuator input commands in Eq. (27) can also be written as

$$
\begin{aligned}
& \bar{u}_{\alpha}^{J}=\left[\begin{array}{ll}
0 & \bar{G}_{\alpha}^{J J}
\end{array}\right]\left\{\begin{array}{l}
x_{\alpha}^{I} \\
x_{\alpha}^{J}
\end{array}\right\} \equiv \bar{G}_{\alpha}^{J} x_{\alpha} \\
& \bar{u}_{\beta}^{J}=\left[\begin{array}{ll}
0 & \bar{G}_{\beta}^{J J}
\end{array}\right]\left\{\begin{array}{l}
x_{\beta}^{I} \\
x_{\beta}^{J}
\end{array}\right\} \equiv \bar{G}_{\beta}^{J} x_{\beta}
\end{aligned}
$$

It is necessary to show that the introduction of augmented joint actuator commands does not affect stability of the closed-loop system. In physical coordinates, the modified $\alpha$-substructure state equation (i.e., $\dot{x}_{\alpha}=A_{\alpha m} x_{\alpha}+B_{\alpha m}^{I} u_{\alpha}^{I}$ ) including the augmented joint input takes the form

$$
\begin{aligned}
&\left\{\begin{array}{c}
\dot{w}_{\alpha}^{I} \\
\ddot{w}_{\alpha}^{I} \\
\cdots \\
\dot{w}_{\alpha}^{J} \\
\ddot{w}_{\alpha}^{J}
\end{array}\right\}=\left[\begin{array}{ccccc}
0 & I & \vdots & 0 & 0 \\
\times & \times & \vdots & \times & \times \\
\cdots & \cdots & \cdots & \cdots \\
0 & 0 & \vdots & 0 & I \\
0 & 0 & \vdots & 0 & 0
\end{array}\right]\left\{\begin{array}{c}
w_{\alpha}^{I} \\
\dot{w}_{\alpha}^{I} \\
\cdots \\
w_{\alpha}^{J} \\
\dot{w}_{\alpha}^{J}
\end{array}\right\}+\left[\begin{array}{c}
0 \\
\times \\
\cdots \\
0 \\
0
\end{array}\right] u_{\alpha}^{I}+\left[\begin{array}{c}
0 \\
\times \\
\cdots \\
0 \\
\times
\end{array}\right] \bar{u}_{\alpha}^{J} \\
& \dot{x}_{\alpha}
\end{aligned}
$$

in which $\times$ denotes those partitions that are in general not zero. The above form is obtained from identifying nonzero terms in the open-loop $A_{\alpha}, B_{\alpha}^{I}, B_{\alpha}^{J}$, and $C_{\alpha}^{J}$ matrices (in physical coordinates) and by using the definitions in Eq. (20). Equation (29) clearly shows that the joint degrees-of-freedom are not controllable by the interior actuators, $u_{\alpha}^{I}$. It also shows that the augmented joint input, $\bar{u}_{\alpha}^{J}$, given in Eq. (27) will not affect stability 
of the closed-loop system, because of the zeros in the lower-left quarter of $A_{\alpha m}$ matrix. The closed-loop system poles are the union of eigenvalues of $\left(A_{\alpha m}^{I I}+B_{\alpha m}^{I I} G_{\alpha}^{I I}\right)$ and the closed-loop poles associated with joint degrees-of-freedom, i.e., $-\zeta_{i} \Omega_{i} \pm-j \Omega_{i} \sqrt{1-\zeta_{i}^{2}}$, $i=1,2, \ldots, n_{c}$. However, Eq. (29) also shows that dynamics of the interior degrees-offreedom are affected by the joint degrees-of-freedom, because of the nonzeros in the upperright quarter of $A_{\alpha m}$ matrix and the nonzero in the upper part of the $B_{\alpha}^{J}$. This interaction, although does not affect stability, can degrade the closed-loop system performance. In order to achieve satisfactory closed-loop performance, the design parameters $\zeta_{i}$ and $\Omega_{i}$ should be assigned such that vibration of joint degrees-of-freedom decays much faster than that of interior degrees-of-freedom.

In summary, the procedure for designing decentralized controller gain matrix consists of three steps. First, the feedback commands for interior actuators are set to be $u_{\alpha}^{I}=G_{\alpha}^{I} x_{\alpha} \equiv\left[\begin{array}{ll}G_{\alpha}^{I I} & 0\end{array}\right] x_{\alpha}$ and $u_{\beta}^{I}=G_{\beta}^{I} x_{\beta} \equiv\left[\begin{array}{cc}G_{\beta}^{I I} & 0\end{array}\right] x_{\beta}$ with $G_{\alpha}^{I I}$ and $G_{\beta}^{I I}$ designed based on $\dot{x}_{\alpha}^{I}=A_{\alpha m}^{I I} x_{\alpha}^{I}+B_{\alpha m}^{I I} u_{\alpha}^{I}$ and $\dot{x}_{\beta}^{I}=A_{\beta m}^{I I} x_{\beta}^{I}+B_{\beta m}^{I I} u_{\beta}^{I}$. Second, the feedback commands for the joint actuators are set to be $u_{\alpha}^{J}=\left(G_{\alpha}^{J}+\bar{G}_{\alpha}^{J}\right) x_{\alpha}$ and $u_{\beta}^{J}=\left(G_{\beta}^{J}+\bar{G}_{\beta}^{J}\right) x_{\beta}$, where $G_{\alpha}^{J}$ and $G_{\beta}^{J}$ are defined in Eq. (23) and $\tilde{G}_{\alpha}^{J}$ and $\bar{G}_{\beta}^{J}$ are defined in Eqs. (27) and (28). This set of feedback commands can be expressed by one equation:

$$
\left\{\begin{array}{c}
u_{\alpha}^{I} \\
u_{\alpha}^{J} \\
u_{\beta}^{J} \\
u_{\beta}^{I}
\end{array}\right\}=\left[\begin{array}{cc}
G_{\alpha}^{I} & 0 \\
G_{\alpha}^{J}+\bar{G}_{\alpha}^{J} & 0 \\
0 & G_{\beta}^{J}+\bar{G}_{\beta}^{J} \\
0 & G_{\beta}^{I}
\end{array}\right]\left\{\begin{array}{l}
x_{\alpha} \\
x_{\beta}
\end{array}\right\} \quad \text { or } \quad \tilde{u}=\tilde{G} \tilde{x}
$$

Finally, after the two substructures are connected together, a global control gain matrix for the global (or assembled) structure can be obtained by substituting Eq. (30) into Eq. (15). The result is

$$
u=T_{1} \tilde{u}=T_{1} \tilde{G} \tilde{x} \equiv G \tilde{x}
$$

The closed-loop poles associated with the joint degrees-of-freedom are not changed by the interconnection of substructures. This is proved by substituting $u^{J}=u_{\alpha}^{J}+u_{\beta}^{J}$ into Eq. (9), which yields $y^{J} \equiv \ddot{w}^{J}=-\left[2 \zeta_{i} \Omega_{i}\right] \dot{w}^{J}-\left[\Omega_{i}^{2}\right] w^{J}$. The set of feedback commands derived in this section will be called the Joint Decoupling Actuator Commands, because they decouple the joints from the interior degrees-of-freedom.

\section{The Design of Decentralized Observers}

In most practical situations, the number of sensors is limited and thus the full measurement of the states is not available. In order to implement state feedback control, an 
observer is required to reconstruct the entire system state. In this section, the procedure for designing decentralized observers is presented.

The derivation is again based on the coupled substructure state-space model in Eq. (10), whose short notation expression is given by Eq. (11). Let the observer be described by

$$
\dot{\tilde{q}}=\tilde{A} \tilde{q}+\tilde{B} \tilde{u}-\tilde{F}(\tilde{y}-\tilde{C} \tilde{q}-\tilde{D} \tilde{u})
$$

where $\tilde{q} \equiv\left\{\begin{array}{l}q_{\alpha} \\ q_{\beta}\end{array}\right\}$ is the observer state vector with $q_{\alpha}$ estimates $x_{\alpha}$ and $q_{\beta}$ estimates $x_{\beta}$, respectively. $\tilde{F}$ is the observer gain matrix to be designed. According to Eq. (30), state feedback using estimated states is done by setting

$$
\tilde{u}=\tilde{G} \tilde{q}=\left[\begin{array}{cc}
G_{\alpha}^{I} & 0 \\
G_{\alpha}^{J}+\bar{G}_{\alpha}^{J} & 0 \\
0 & G_{\beta}^{J}+\bar{G}_{\beta}^{J} \\
0 & G_{\beta}^{I}
\end{array}\right]\left\{\begin{array}{l}
q_{\alpha} \\
q_{\beta}
\end{array}\right\}
$$

Substituting Eq. (33) into the observer equation (32) and performing algebraic manipulation, we get

$$
\begin{aligned}
& \left\{\begin{array}{l}
\dot{q}_{\alpha} \\
\dot{q}_{\beta}
\end{array}\right\}=\left[\begin{array}{cc}
A_{\alpha m}+B_{\alpha m}^{I} G_{\alpha}^{I} & 0 \\
0 & A_{\beta m}+B_{\beta m}^{I} G_{\beta}^{I}
\end{array}\right]\left\{\begin{array}{l}
q_{\alpha} \\
q_{\beta}
\end{array}\right\}+\left\{\begin{array}{l}
B_{\alpha}^{J} S^{-1} D_{\beta}^{J J} \\
B_{\beta}^{J} S^{-1} D_{\alpha}^{J J}
\end{array}\right\} \bar{u}^{J} \\
& +\tilde{F}\left(\left[\begin{array}{cc}
C_{\alpha m}^{I}+D_{\alpha m}^{I I} G_{\alpha}^{I} & 0 \\
0 & 0 \\
0 & 0 \\
0 & C_{\beta m}^{I}+D_{\beta m}^{I I} G_{\beta}^{I}
\end{array}\right]\left\{\begin{array}{l}
q_{\alpha} \\
q_{\beta}
\end{array}\right\}+\left\{\begin{array}{l}
D_{\alpha}^{I J} S^{-1} D_{\beta}^{J J} \\
D_{\alpha}^{J J} S^{-1} D_{\beta}^{J J} \\
D_{\alpha}^{J J} S^{-1} D_{\beta}^{J J} \\
D_{\beta}^{I J} S^{-1} D_{\alpha}^{J J}
\end{array}\right\} \bar{u}^{J}-\left\{\begin{array}{l}
y_{\alpha}^{I} \\
y_{\alpha}^{J} \\
y_{\beta}^{J} \\
y_{\beta}^{I}
\end{array}\right\}\right)
\end{aligned}
$$

where $\bar{u}^{J}=\left(\bar{G}_{\alpha}^{J} q_{\alpha}+\bar{G}_{\beta}^{J} q_{\beta}\right)$ and

$$
\begin{array}{lll}
C_{\alpha m}^{I}=C_{\alpha}^{I}-D_{\alpha}^{I J}\left(D_{\alpha}^{J J}\right)^{-1} C_{\alpha}^{J} & , & D_{\alpha m}^{I I}=D_{\alpha}^{I I}-D_{\alpha}^{I J}\left(D_{\alpha}^{J J}\right)^{-1} D_{\alpha}^{J I} \\
C_{\beta m}^{I}=C_{\beta}^{I}-D_{\alpha}^{I J}\left(D_{\beta}^{J J}\right)^{-1} C_{\beta}^{J} & , & D_{\beta m}^{I I}=D_{\beta}^{I I}-D_{\beta}^{I J}\left(D_{\beta}^{J J}\right)^{-1} D_{\beta}^{J I}
\end{array}
$$

The above matrices in addition to the $A_{\alpha m}, A_{\beta m}, B_{\alpha m}^{I}$, and $B_{\beta m}^{I}$ matrices given in Eq. (20) completely define the modified substructure state-space models.

Equation (34) indicates that a decentralized observer design can be achieved by appropriate choice of observer gain matrix. First, restrict the observer gain matrix $\tilde{F}$ to take the form

$$
\tilde{F}=\left[\begin{array}{cccc}
F_{\alpha}^{I} & F_{\alpha}^{J} & 0 & 0 \\
0 & 0 & F_{\beta}^{J} & F_{\beta}^{I}
\end{array}\right]
$$


which is similar to the form of the controller gain matrix $\tilde{G}$ in Eq. (30). Then, set the joint output observer gain matrices to be

$$
\begin{aligned}
& F_{\alpha}^{J}=-\left(B_{\alpha}^{J}+F_{\alpha}^{I} D_{\alpha}^{I J}\right)\left(D_{\alpha}^{J J}\right)^{-1} \\
& F_{\beta}^{J}=-\left(B_{\beta}^{J}+F_{\beta}^{I} D_{\beta}^{I J}\right)\left(D_{\beta}^{J J}\right)^{-1}
\end{aligned}
$$

Substitution of Eqs. (36) and (37) into the observer equation (34) gives

$$
\begin{aligned}
& \dot{q}_{\alpha}=\left(A_{\alpha m}+B_{\alpha m}^{I} G_{\alpha}^{I}+F_{\alpha}^{I} C_{\alpha m}^{I}+F_{\alpha}^{I} D_{\alpha m}^{I I} G_{\alpha}^{I}\right) q_{\alpha}-F_{\alpha}^{I} y_{\alpha}^{I}-F_{\alpha}^{J} y_{\alpha}^{J} \\
& \dot{q}_{\beta}=\left(A_{\beta m}+B_{\beta m}^{I} G_{\beta}^{I}+F_{\beta}^{I} C_{\beta m}^{I}+F_{\beta}^{I} D_{\beta m}^{I I} G_{\beta}^{I}\right) q_{\beta}-F_{\beta}^{I} y_{\beta}^{I}-F_{\beta}^{J} y_{\beta}^{J}
\end{aligned}
$$

Or, in short notation,

$$
\begin{aligned}
& \dot{q}_{\alpha}=E_{\alpha} q_{\alpha}-\left[\begin{array}{ll}
F_{\alpha}^{I} & F_{\alpha}^{J}
\end{array}\right] y_{\alpha} \\
& \dot{q}_{\beta}=E_{\beta} q_{\beta}-\left[\begin{array}{ll}
F_{\beta}^{I} & F_{\beta}^{J}
\end{array}\right] y_{\beta}
\end{aligned}
$$

where the definitions of $E_{\alpha}$ and $E_{\beta}$ are obvious. Therefore, by restricting the observer gain matrix to have a decentralized form as in Eq. (36) and by setting the joint output observer gain matrices as in Eq. (37), the observer equation is decoupled. The interior output observer gain matrices $F_{\alpha}^{I}$ and $F_{\beta}^{I}$ still need to be designed. Define a state estimation error vector as

$$
\tilde{e} \equiv\left\{\begin{array}{l}
e_{\alpha} \\
e_{\beta}
\end{array}\right\} \equiv\left\{\begin{array}{l}
x_{\alpha}-q_{\alpha} \\
x_{\beta}-q_{\beta}
\end{array}\right\}
$$

Then, after algebraic manipulations, subtraction of the coupled substructure state equation (11) from the observer equation (32) yields

$$
\begin{aligned}
& \dot{e}_{\alpha}=A_{\alpha m} e_{\alpha}+F_{\alpha}^{I} C_{\alpha m}^{I} e_{\alpha} \\
& \dot{e}_{\beta}=A_{\beta m} e_{\beta}+F_{\beta}^{I} C_{\beta m}^{I} e_{\beta}
\end{aligned}
$$

The above error dynamic equation is decoupled. Any existing pole placement algorithm can be used to determine $F_{\alpha}^{I}$ and $F_{\beta}^{I}$. In order for the state reconstruction to be effective for feedback, $F_{\alpha}^{I}$ and $F_{\beta}^{I}$ should be designed such that eigenvalues of $\left(A_{\alpha m}+\right.$ $\left.F_{\alpha}^{I} C_{\alpha m}\right)$ and $\left(A_{\beta m}+F_{\beta}^{I} C_{\beta m}^{I}\right)$ are further to the left in the complex plane than the regulator poles, which, as mentioned previously, are the union of eigenvalues of $\left(A_{\alpha m}^{I I}+B_{\alpha m}^{I I} G_{\alpha}^{I I}\right)$ and $\left(A_{\beta m}^{I I}+B_{\beta m}^{I I} G_{\beta}^{I I}\right)$ and the closed-loop poles associated with joint degrees-of-freedom, i.e., $-\zeta_{i} \Omega_{i} \pm j \Omega_{i} \sqrt{1-\zeta_{i}^{2}}, i=1,2, \ldots, n_{c}$. Of course, we have made an assumption that both $\left(A_{\alpha m}, C_{\alpha m}^{I}\right)$ and $\left(A_{\beta m}, C_{\beta m}^{I}\right)$ are completely observable. This assumption is by no means a restriction. In practice, it is usually possible to arrange locations of interior sensors to meet this assumption. Observability of the modified substructure state-space models and optimization of substructure interior sensor locations are future research topics. 
Although the derivation so far has been based on a two-component structure, extension of the method to multi-component structure is straightforward. The controller formed by the substructure observers and the Joint Decoupling Actuator Commands will be called the Joint Decoupling Controller. In summary, the design procedure for Joint Decoupling Controllers can be described by the following steps.

(1) Determine substructure state-space models

$$
\begin{gathered}
\dot{x}_{s}=A_{s} x_{s}+\left[\begin{array}{ll}
B_{s}^{I} & B_{s}^{J}
\end{array}\right]\left\{\begin{array}{c}
u_{s}^{I} \\
u_{s}^{J}
\end{array}\right\} \\
\left\{\begin{array}{c}
y_{s}^{I} \\
y_{s}^{J}
\end{array}\right\}=\left[\begin{array}{c}
C_{s}^{I} \\
C_{s}^{J}
\end{array}\right] x_{s}+\left[\begin{array}{cc}
D_{s}^{I I} & D_{s}^{I J} \\
D_{s}^{J I} & D_{s}^{J J}
\end{array}\right]\left\{\begin{array}{c}
u_{s}^{I} \\
u_{s}^{J}
\end{array}\right\}
\end{gathered}
$$

from finite-element modeling or from system identification of the substructures.

(2) Derive the modified substructure state-space models

$$
\begin{aligned}
& \dot{x}_{s}=A_{s m} x_{s}+B_{s m}^{I} u_{s}^{I} \\
& y_{s}^{I}=C_{s m}^{I} x_{s}+D_{s m}^{I I} u_{s}^{I}
\end{aligned} \quad s=\alpha, \beta, \gamma, \ldots
$$

by using Eqs. (20) and (35).

(3) Design substructure control gains:

(a) Design $G_{s}^{I I}$ based on the modified substructure subsystem $\dot{x}_{s}^{I}=A_{s m}^{I I} x_{s}^{I}+$ $B_{s m}^{I I} u_{s}^{I}$ and then, form $G_{s}^{I}=\left[\begin{array}{ll}G_{s}^{I I} & 0\end{array}\right]$.

(b) Set $G_{s}^{J}=-\left(D_{s}^{J J}\right)^{-1}\left(C_{s}^{J}+D_{s}^{J I} G_{s}^{I}\right)$.

Choose appropriate values of $\zeta_{i}$ and $\Omega_{i}$ for joint degrees-of-freedom and then, form $\bar{G}_{s}^{J}=\left[\begin{array}{ll}0 & \bar{G}^{J J}\end{array}\right]$, where $\bar{G}_{s}^{J J}=\left(D_{s}^{J J}\right)^{-1}\left[-\left[\Omega_{i}^{2}\right]-\left[2 \zeta_{i} \Omega_{i}\right]\right]$.

(c) Set $u_{s}^{I}=G_{s}^{I} q_{s}, \quad u_{s}^{J}=\left(G_{s}^{J}+\bar{G}_{s}^{J}\right) q_{s}$.

(4) Design substructure observers:

(d) Design $F_{s}^{I}$ based on $\left(A_{s m}, C_{s m}^{I}\right)$.

(e) Set $F_{s}^{J}=-\left(B_{s}^{J}+F_{s}^{I} D_{s}^{I J}\right)\left(D_{s}^{J J}\right)^{-1}$.

(f) Form substructure observer equation:

$$
\dot{q}_{s}=\left(A_{s m}+B_{s m}^{I} G_{s}^{I}+F_{s}^{I} C_{s m}^{I}+F_{s}^{I} D_{s m}^{I I} G_{s}^{I}\right) q_{s}-F_{s}^{I} y_{s}^{I}-F_{s}^{J} y_{s}^{J}
$$


(5) From global feedback gain matrix for the global structure by using the input coupling matrix $T_{1}$, i.e., using the relation $u=T_{1} \tilde{u}$ (see Eqs. (30) and (31)).

It is emphasized that although the substructure control gain matrices need to be assembled (Step (5)) for implementation on the global (or assembled) structure, there is no need to assemble the substructure observers. In fact, we leave the substructure observers unassembled so that the control implementation is decentralized. However, it should be pointed out that in actual implementation, $y_{\alpha}^{J}$ and $y_{\beta}^{J}$ in the substructure observer equations (38) are replaced by $y^{J}$, since, according to compatibility condition, $y_{\alpha}^{J}=y_{\beta}^{J}=y^{J}$. The closed-loop system of the two-component structure, obtained by combining the global structure state-space model in Eq. (12), the feedback law given in Eq. (33), and the substructure observers in Eq. (39), and by using the input and output coupling matrices, is given by

$$
\left\{\begin{array}{c}
\dot{x} \\
\dot{\tilde{q}}
\end{array}\right\}=\left[\begin{array}{cc}
A & B T_{1} \tilde{G} \\
-\tilde{F} T_{2} C & \tilde{E}-\tilde{F} T_{2} D T_{1} \tilde{G}
\end{array}\right]\left\{\begin{array}{l}
x \\
\tilde{q}
\end{array}\right\}
$$

where $\tilde{E}=\left[\begin{array}{cc}E_{\alpha} & 0 \\ 0 & E_{\beta}\end{array}\right]$.

The above derivation for the substructure observers is based on the "coupled" state-space model in Eq. (10). Therefore, as shown in the derivation the collection of substructure observers together with the global feedback gain matrix (i.e., $T_{1} \tilde{G}$ ) form a stabilizing controller for the global structure. However, it is easy to show that each individual substructure observer together with substructure feedback gain matrix also constitute a stabilizing controller for corresponding individual substructures. To be more precise, define $\alpha$-substructure control gain and observer gain matrices to be

$$
G_{\alpha} \equiv\left\{\begin{array}{c}
G_{\alpha}^{I} \\
G_{\alpha}^{J}+\bar{G}_{\alpha}^{J}
\end{array}\right\} \quad, \quad F_{\alpha} \equiv\left[\begin{array}{ll}
F_{\alpha}^{I} & F_{\alpha}^{J}
\end{array}\right]
$$

Then, by combining the "unconnected" $\alpha$-substructure model in Eq. (1), the $\alpha$-observer in Eq. (38a) and the feedback law $u_{\alpha}^{I}=G_{\alpha}^{I} q_{\alpha}$ and $u_{\alpha}^{J}=\left(G_{\alpha}^{J}+\bar{G}_{\alpha}^{J}\right) q_{\alpha}$, we get the following closed-loop equation

$$
\left\{\begin{array}{c}
\dot{x}_{\alpha} \\
\dot{q}_{\alpha}
\end{array}\right\}=\left[\begin{array}{cc}
A_{\alpha} & B_{\alpha} G_{\alpha} \\
-F_{\alpha} C_{\alpha} & E_{\alpha}-F_{\alpha} D_{\alpha} G_{\alpha}
\end{array}\right]\left\{\begin{array}{c}
x_{\alpha} \\
q_{\alpha}
\end{array}\right\}
$$

Using the state estimation error vector defined previously, the above equation can be converted to

$$
\left\{\begin{array}{c}
\dot{x}_{\alpha} \\
\dot{e}_{\alpha}
\end{array}\right\}=\left[\begin{array}{cc}
A_{\alpha m}+B_{\alpha m}^{I} G_{\alpha}^{I}+B_{\alpha}^{J} \bar{G}_{\alpha}^{J} & -B_{\alpha} G_{\alpha} \\
0 & A_{\alpha m}+F_{\alpha}^{I} C_{\alpha m}^{I}
\end{array}\right]\left\{\begin{array}{l}
x_{\alpha} \\
e_{\alpha}
\end{array}\right\}
$$


which obviously is a stable system. The closed-loop poles are the same as those of the $\alpha$-substructure when it is connected to $\beta$. Therefore, substructure's closed-loop poles are the same before and after the connection. Besides that, each substructure's closedloop response is affected by the connection only to a slight degree. If exact states are available for feedback (which implies the case of full state measurement), any pair of connecting joint degrees-of-freedom will have the same response before and after connection, providing that before connection the initial conditions of separate joints are the same. Consequently, each substructure's closed-loop responses is the same whether the substructures are connected or not. When estimate state is used for feedback, because the joint degrees-of-freedom are set to have fast decaying dynamics, the responses of interior degrees-of-freedom will be affected by the interconnection only slightly. This feature makes the proposed Joint Decoupling Controllers attractive for active control of space structures that are required to be connected and disconnected on a routine basis. No controller redesign or controller shut-off-and-turn-on is necessary before, during, and after the entire connecting process.

If the dimensions of substructures are still too large for control design purposes, model reduction can be performed to substructure state-space models. Then, a loworder Joint Decoupling Controller can be designed for each substructure based on the reduced-order substructure model. It certainly is easier to perform model reduction at the substructure level than at the global structure level. Although model reduction introduces the so-called "spillover" problem, the closed-loop system of the global structure is stable as long as all the substructure closed-loop systems are stable.

\section{Examples}

\section{A. A Mass-Spring-Damper Example}

The first example is the two-component mass-spring-damper system shown in Fig. 2. The two substructures $\alpha$ and $\beta$ are to be connected by using the rigid links on mass (4) and mass (5). Displacement coordinates of the global structure and the two substructures are designated by $w_{i}, w_{\alpha i}$, and $w_{\beta i}$, respectively. The outputs of the $\alpha$-substructure are: $y_{\alpha}^{I}=w_{\alpha 1}$ and $y_{\alpha}^{J}=\ddot{w}_{\alpha 4}$. The outputs of the $\beta$-substructure are: $y_{\beta}^{J}=\ddot{w}_{\beta 3}$ and $y_{\beta}^{I}=w_{\beta 1}$. All the inputs are forces.

The method in this paper is used to design state feedback gain matrix for the two substructures. Then, the substructure state feedback gain matrices are assembled to form a global state feedback gain matrix for the global structure. The local feedback gain 

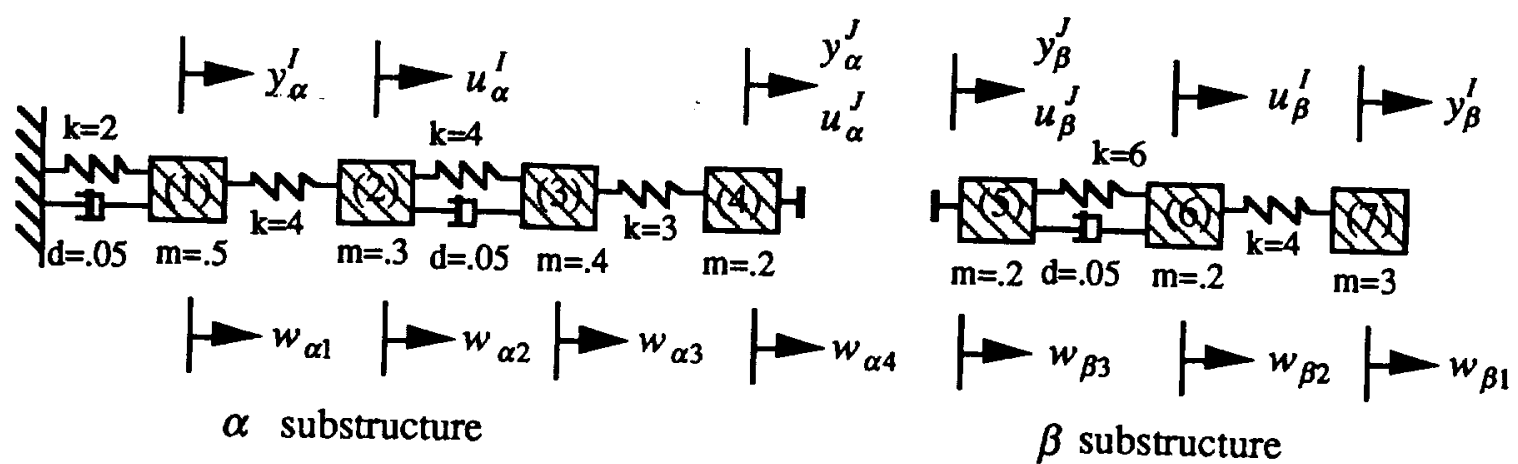

$\beta$ substructure

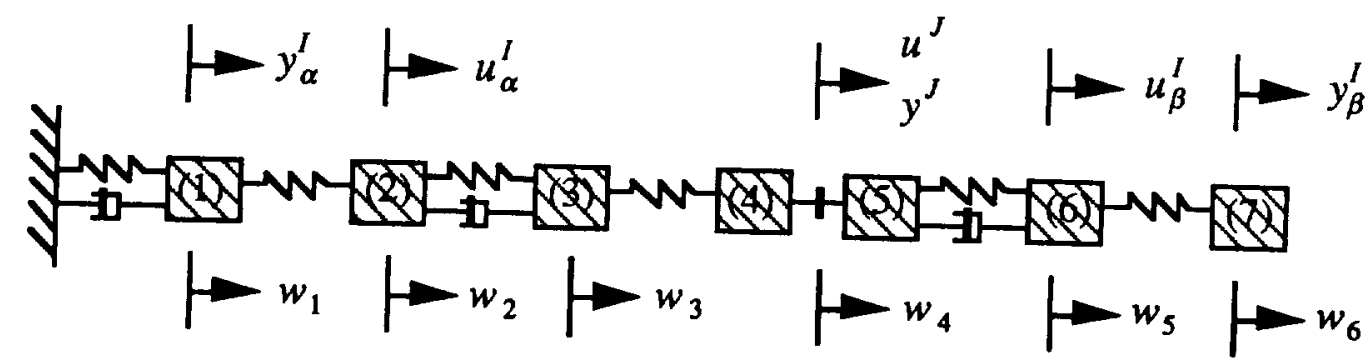

The global structure

Figure 2: A mass-spring-damper example.

matrix $G_{\alpha}^{I I}$ and $G_{\beta}^{I I}$ for the interior actuators are determined by solving the following optimization problems:

$$
\begin{aligned}
& \text { Min. } J_{\alpha}=\int_{0}^{\infty}\left(x_{\alpha}^{I}\right)^{T} Q_{\alpha}^{I I} x_{\alpha}^{I}+\rho\left(u_{\alpha}^{I}\right)^{T} u_{\alpha}^{I} d t \quad \text { subj. to } \dot{x}_{\alpha}^{I}=A_{\alpha m}^{I I} x_{\alpha}^{I}+B_{\alpha m}^{I I} u_{\alpha}^{I} \\
& \text { Min. } J_{\beta}=\int_{0}^{\infty}\left(x_{\beta}^{I}\right)^{T} Q_{\beta}^{I I} x_{\beta}^{I}+\rho\left(u_{\beta}^{I}\right)^{T} u_{\beta}^{I} d t \quad \text { subj. to } \dot{x}_{\beta}^{I}=A_{\beta m}^{I I} x_{\beta}^{I}+B_{\alpha m}^{I I} u_{\beta}^{I}
\end{aligned}
$$

The regulation cost weighting matrices were chosen to be

$$
Q_{\alpha}^{I I}=\left[\begin{array}{cc}
K_{\alpha}^{I I} & 0 \\
0 & M_{\alpha}^{I I}
\end{array}\right] \quad, \quad Q_{\beta}^{I I}=\left[\begin{array}{cc}
K_{\beta}^{I I} & 0 \\
0 & M_{\beta}^{I I}
\end{array}\right]
$$

such that the first terms in the integrals represent the sum of strain energy and kinetic energy corresponding to the interior degrees-of-freedom of the substructures. The control cost weight parameter $\rho$ was set to be 0.1 . The design parameters for the augmented joint actuator commands were chosen to be $\Omega^{2}=25$ and $2 \zeta \Omega=8$ such that the closed-loop poles corresponding to the joint degree-of-freedom are $-4 \pm j 3$. For each substructure, an observer is also designed according to the proposed decentralized observer design procedure. The observer gain matrices are determined by using a pole placement method such that observer poles are 12 units to the left of regulator poles in the complex plane. 
The results are summarized in Tables 1 and 2 and Figs. 4 through 7. Table 1 lists the control gain and observer gain matrices of each substructure. The global control gain matrix for the global structure is simply an assemblage of substructure control gain matrices. Table 2 lists the closed-loop system poles. It is seen that each substructure has a pair of regulator poles $-4 \pm j 3$ associated with the joint degree-of-freedom. The global structure regulator poles are the union of substructure regulator poles, except that one pair of $-4 \pm j 3$ are missing because the substructure joints have been connected to form the global structure. The substructure observer poles are equal to regulator poles minus 12 as the design specification required. The global structure observer poles are the union of substructure observer poles.

Assume the global structure is impacted by a unit impulse force on the joint degreeof-freedom at time zero. This impact force gives the joint degree-of-freedom an initial velocity of magnitude 2.5. Thus, the initial state of the substructure is given by $x_{0}=$ $[0,0,0,0,0,0,0,0,0,2.5,0,0]$. In practical situations, the initial state is unknown. Therefore, the initial state of the observer is set to be zero, i.e., $q_{0}=[0,0,0,0,0,0,0,0,0,0,0,0]$. Based on these initial conditions, the global structure open-loop and closed-loop displacement response histories at $w_{1}, w_{2}$, and $w_{3}$ degrees-of-freedom are calculated and shown in Fig. 4 and Fig. 5, respectively. It is seen the proposed Joint Decoupling Controllers successfully suppressed the vibration of the structure. In Section IV, we mentioned that the Joint Decoupling Controllers also operate well at individual substructure level. This is demonstrated by Figs. 6 and 7. Figure 6 shows the closed-loop response histories of the $\alpha$-substructure (for the same initial conditions) when it is disconnected from the $\beta$-substructure. Figure 7 compares in an expanded axis scale the closed-loop response of DOF1 of the global structure and the closed-loop response of DOF1 of the "disconnected" $\alpha$-substructure. It is evident that the system response is only slightly affected by the interconnection of substructures.

\section{B. A Three-Truss Structure Example}

The second example is the two-dimensional three-truss structure shown in Fig. 3. The three truss components are to be connected at the joints, at which there are collocated actuators and accelerometers. Each substructure has two interior actuators located at arrows designated with " $a$ ". The initial conditions is induced by an impact force of magnitude 1000 applied at the lower-right corner of $\alpha$ in the $x$-direction.

A set of Joint Decoupling Actuator Commands are designed by using the proposed procedure. The interior actuator gain matrices are determined by the LQR theory following the formulation in the Mass-spring-damper Example. The augmented joint ac- 


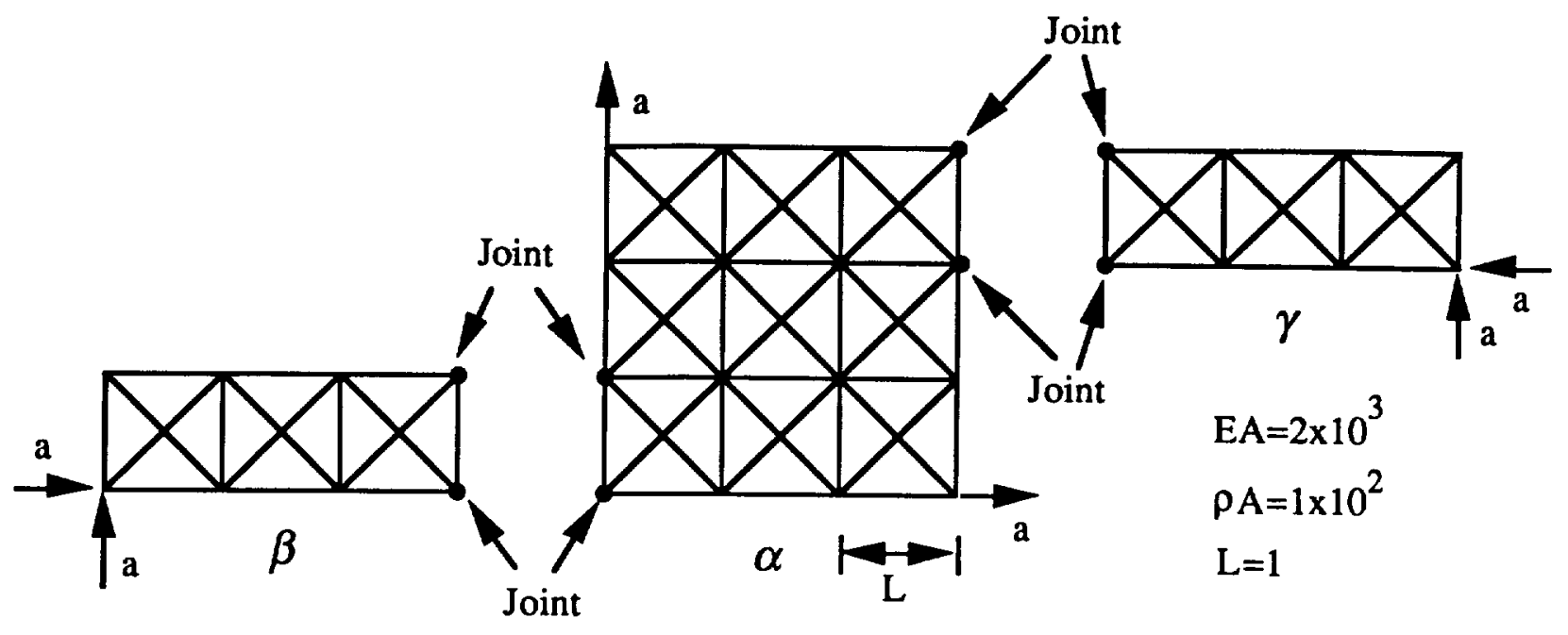

Figure 3: A three-truss structure example

tuator commands are also set to be such that all joint degrees-of-freedom have the same closed-loop poles: $-4 \pm j 3$. Observer design is not included in this example because pole placement calculation exceeded computer capacity. (All the computations were done on a Macintosch IIci computer using MATLAB software.) Therefore, exact state feedback is used.

The design results are shown in Figs. 8 through 11. Open-loop displacement response at the lower-right corner of $\alpha$-substructure in the $x$-direction is shown in Fig. 8. The open-loop response diverges because the system has rigid-body motion. Figure 9 shows the closed-loop displacement response histories at the lower-right corner of $\alpha$ substructure. Figure 10 shows the closed-loop displacement histories at the lower-left corner of $\beta$-substructure. Note that the responses in Fig. 10 is of order $10^{-2}$, which is $1 / 100$ of the responses in Fig. 9. Evidently, the vibration of $\beta$-substructure is suppressed by its own interior actuator inputs and is not affected by the vibration of $\alpha$-substructure, because the interaction between $\alpha$ and $\beta$ is eliminated. Figure 11 shows the closed-loop displacement responses of the left joint degrees-of-freedom. Note that the joint responses have magnitudes of order $10^{-3}$ and are suppressed in very short time. Responses of the $\gamma$-substructure are similar and therefore are omitted.

All the closed-loop responses were calculated by using the closed-loop equation of the global structure. Closed-loop responses of each "unconnected" individual substructure for the same given initial conditions were also calculated. They turned out to be exactly the same as the closed-loop responses included here. As discussed at the end of Section IV, each substructure's response is the same whether the substructures are 
connected or not, if exact state feedback is used. This example also demonstrates the computational efficiency of the proposed decentralizd design method. The global structure has 56 DOFs (112 states). A centralized design approach will involve solving a Riccati equation of order 112. By dividing the global structure into three-components one component has $32 \mathrm{DOFs}$, two components have $16 \mathrm{DOFs}$ - the size of the control design problem is significantly reduced.

\section{Concluding Remarks}

A substructure-based control design procedure using the idea of Joint Decoupling has been developed for decentralized control of large complex flexible structures. The method assumes there are collocated actuators and sensors at every joint degree-offreedom. Conditions of compatibility and equilibrium at the interface between substructures are used to derive a coupled substructure state-space model for the structure to be controlled, which leads to modified substructure state-space models to be used as decentralized design basis. The design concept is summarized as follows. The control commands for interior actuators of each substructure are chosen to be localized state feedback. Then, the joint actuator commands are set to cancel out all the forces acting on the joint degrees-of-freedom, so that interactions between substructures are eliminated. Finally, a set of augmented commands are added to the joint actuators to stabilize the joint degrees-of-freedom. The advantages of the proposed method are: (1) the design process is completely decentralized, (2) the controllers can function at both individual substructure and global structure levels, and (3) a large scale control design problem is divided into several small scale subproblems. A six-degree-of-freedom mass-spring-damper system and a three-truss structure are used to demonstrate the proposed method. It shows that the proposed controller successfully suppresses the vibration of the individual substructure as well as the vibration of the global structure.

\section{Acknowledgment}

This research work was done while the first author held a National Research Council Research Associateship at the NASA Langley Research Center.

\section{References}

1. Singh, M. G., Titli, A., and Malinowski, K., "Decentralised Control Design: An Overview," Large Scale Systems, Vol. 9, 1985, pp. 215-230. 
2. Wang, S. H. and Davison, E. J., "On the Stabilization of Decentralized Control Systems," IEEE Trans. Automat. Control, Vol. AC-18, No. 5, 1973, pp. 473-478.

3. Ikeda, M., Šiljak, D. D., and White, D. E., "Decentralized Control with Overlapping Information Sets," J. of Optim. Theory and Appl., Vol. 34, No. 2, 1981, pp. 279-309.

4. West-Vukovich, G. S., Davison, E. J., and Hughes, P. C., "The Decentralized Control of Flexible Space Structures," IEEE Trans. Automat. Control, Vol. AC-29, No. 10, 1984 , pp. $866-878$.

5. K. D. Young, "Distributed Finite-Element Modeling and Control Approach for Large Flexible Structures," J. Guidance, Vol. 13, No. 4, 1990, pp. 703-713.

6. Su, T. J. and Craig, R. R., Jr.,"Substructuring Decomposition and Controller Synthesis," 31st AIAA/ASME/ASCE/AHS/ASC Structures, Structural Dynamics, and Materials Conference, Long Beach, CA, April 2-4, 1990, pp. 1932-1940. 
Table 1: State feedback gain matrices obtained from decentralized control design.

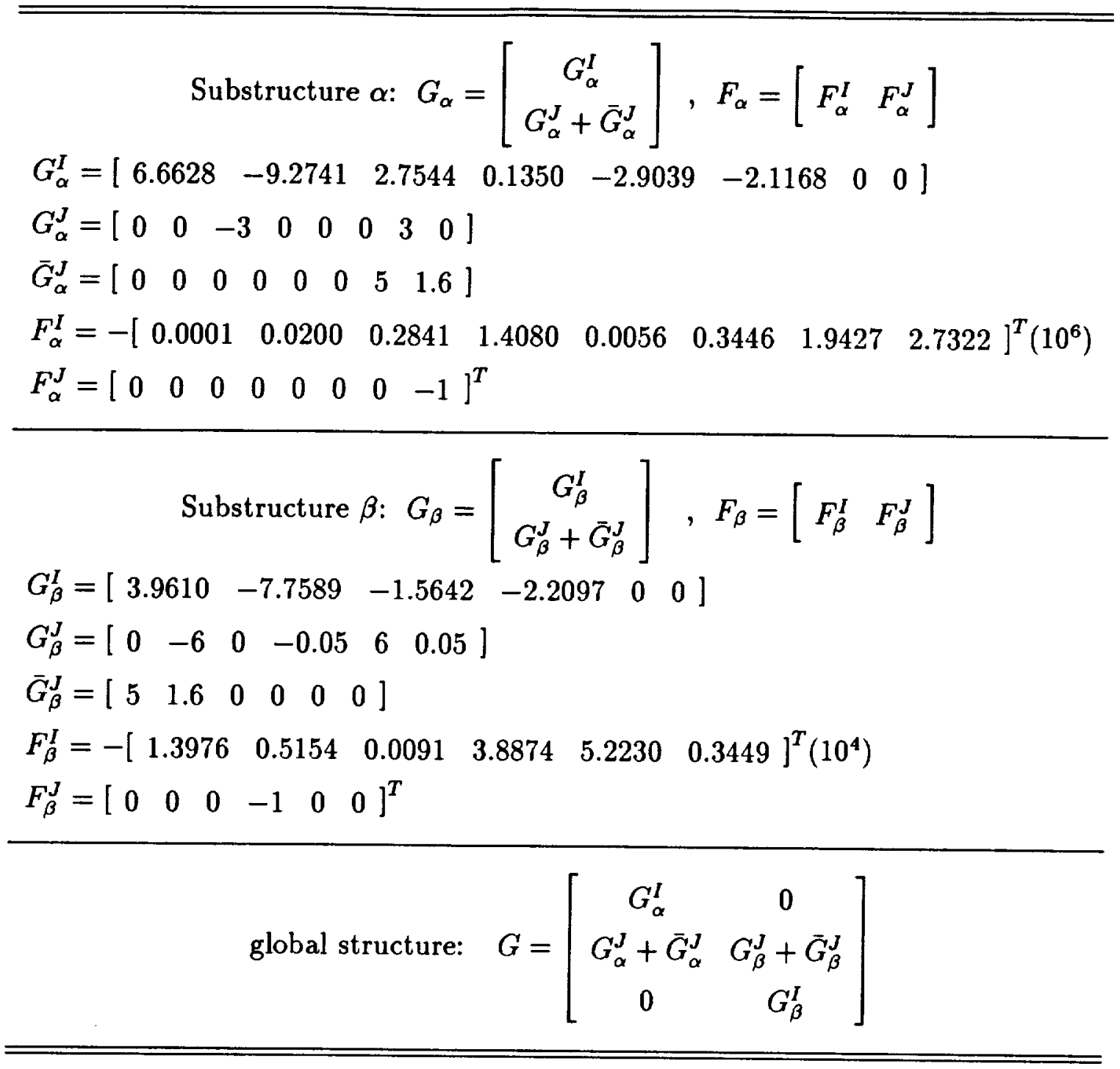


Table 2: Poles of closed-loop systems.

\begin{tabular}{|c|c|}
\hline \multicolumn{2}{|c|}{ Substructure $\alpha$} \\
\hline Regulator Poles & Observer Poles \\
\hline-3.01 & -15 . \\
\hline-0.33 & -12 . \\
\hline-1.6 & -1 \\
\hline$-4 . c$ & -16 \\
\hline \multicolumn{2}{|l|}{ Substr } \\
\hline Regulator Poles & er Poles \\
\hline$-4.4318 \pm j 7.194$ & $\pm j 7.1943$ \\
\hline-1.2 & -13 \\
\hline-4.00 & -16 \\
\hline \multicolumn{2}{|r|}{ ture } \\
\hline Regulator Poles & Observer Poles \\
\hline$-3.0178 \pm j 5.6888$ & $-15.0178 \pm j 5.6888$ \\
\hline$-0.3371 \pm j 3.8531$ & $\pm j 3.8531$ \\
\hline-1.68 & -13. \\
\hline $\pm j 7.1943$ & -16.4 \\
\hline 1 i2 7800 & $-13.2173 \pm j 2.7689$ \\
\hline-4.0 & -16.000 \\
\hline & $-16.0000 \pm j 3$ \\
\hline
\end{tabular}




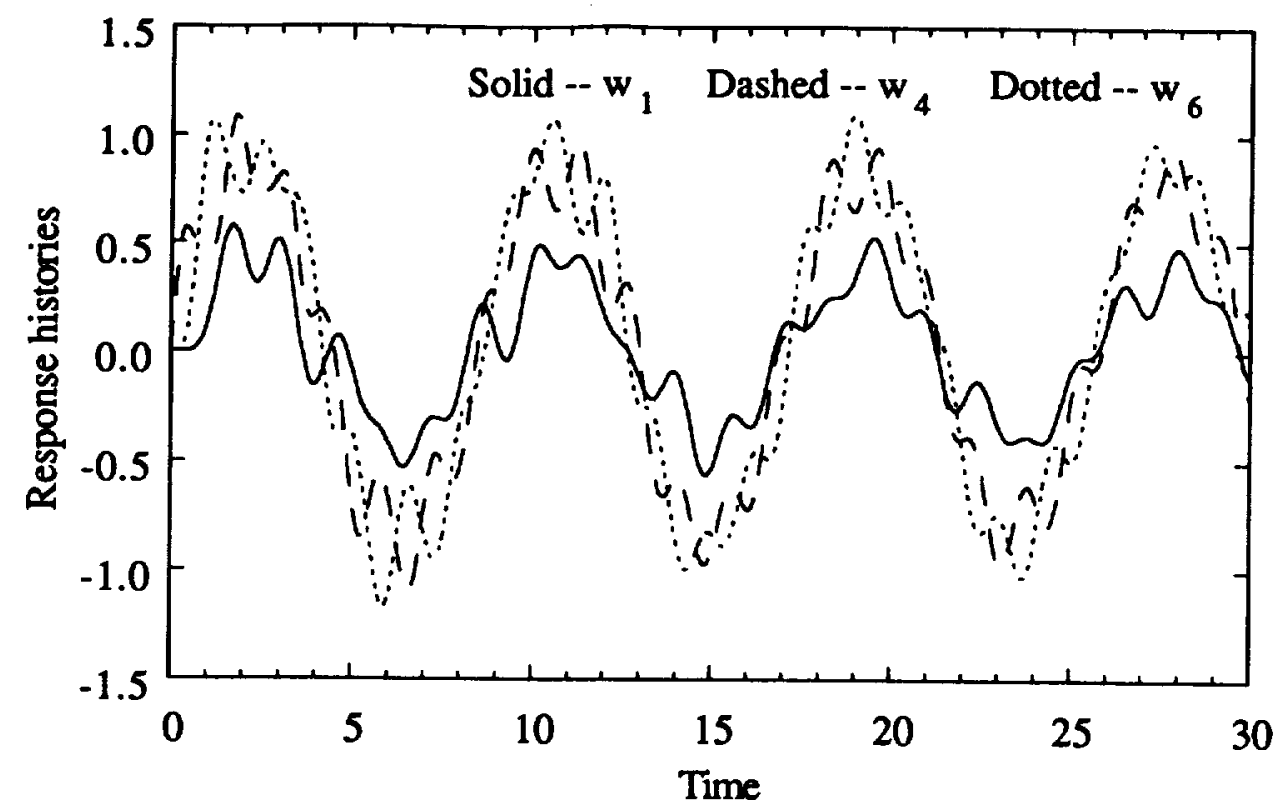

Figure 4: Open-loop response histories of global structure (Mass-spring-damper example).

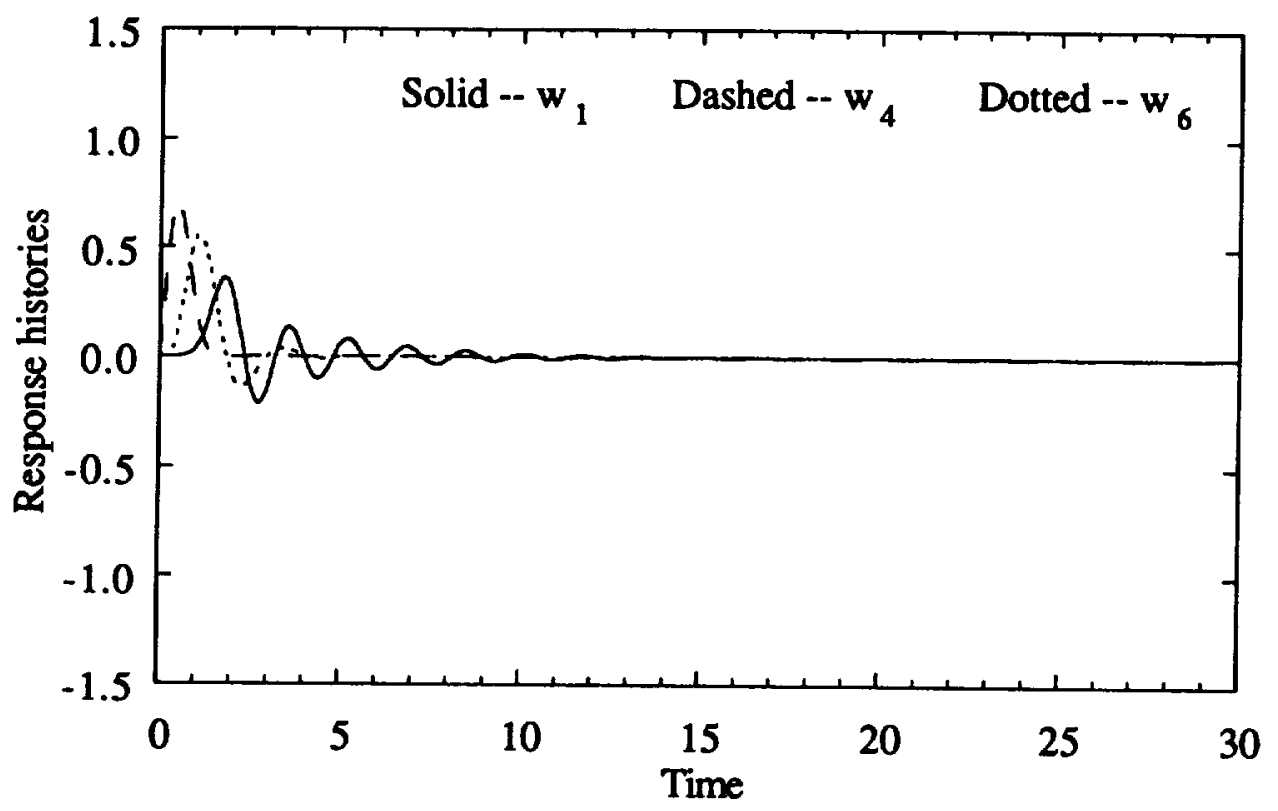

Figure 5: Closed-loop response histories of global structure (Mass-spring-damper example). 


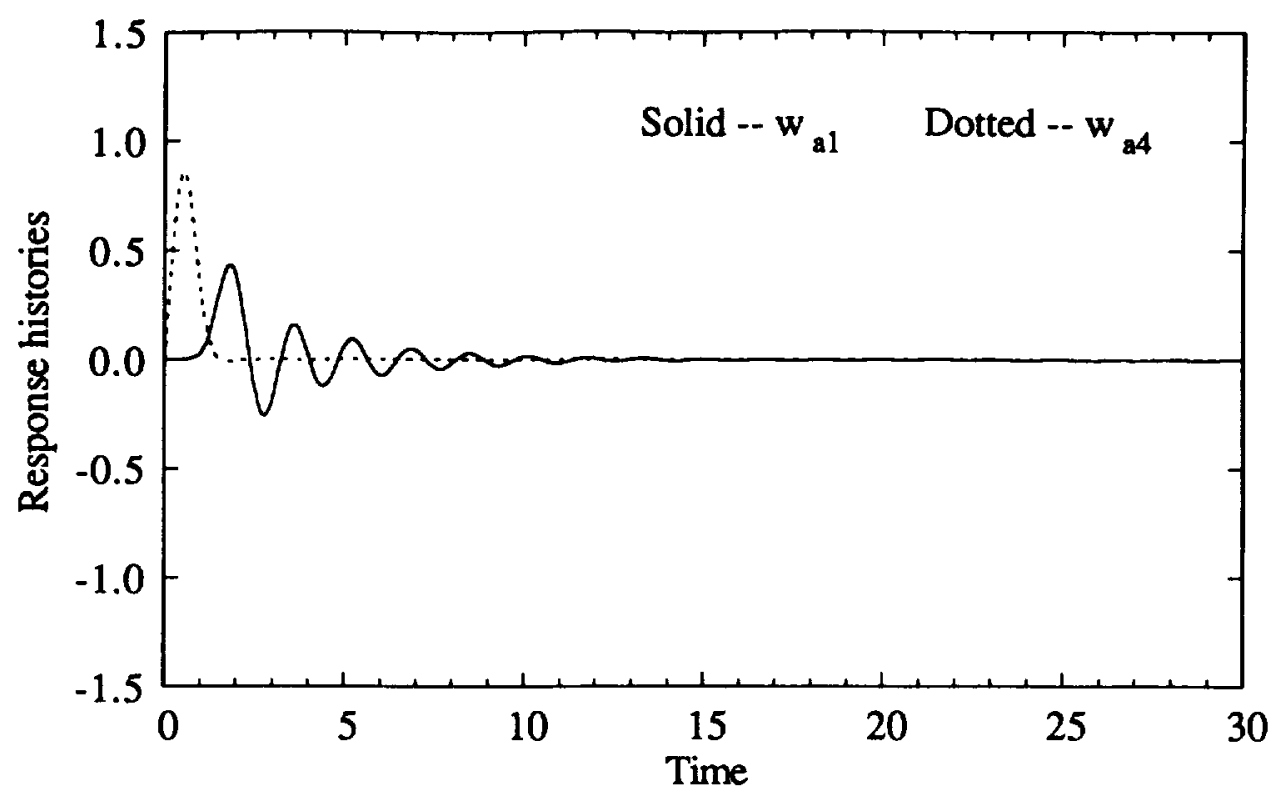

Figure 6: Closed-loop response histories of $\alpha$-substructure (Mass-spring-damper example).

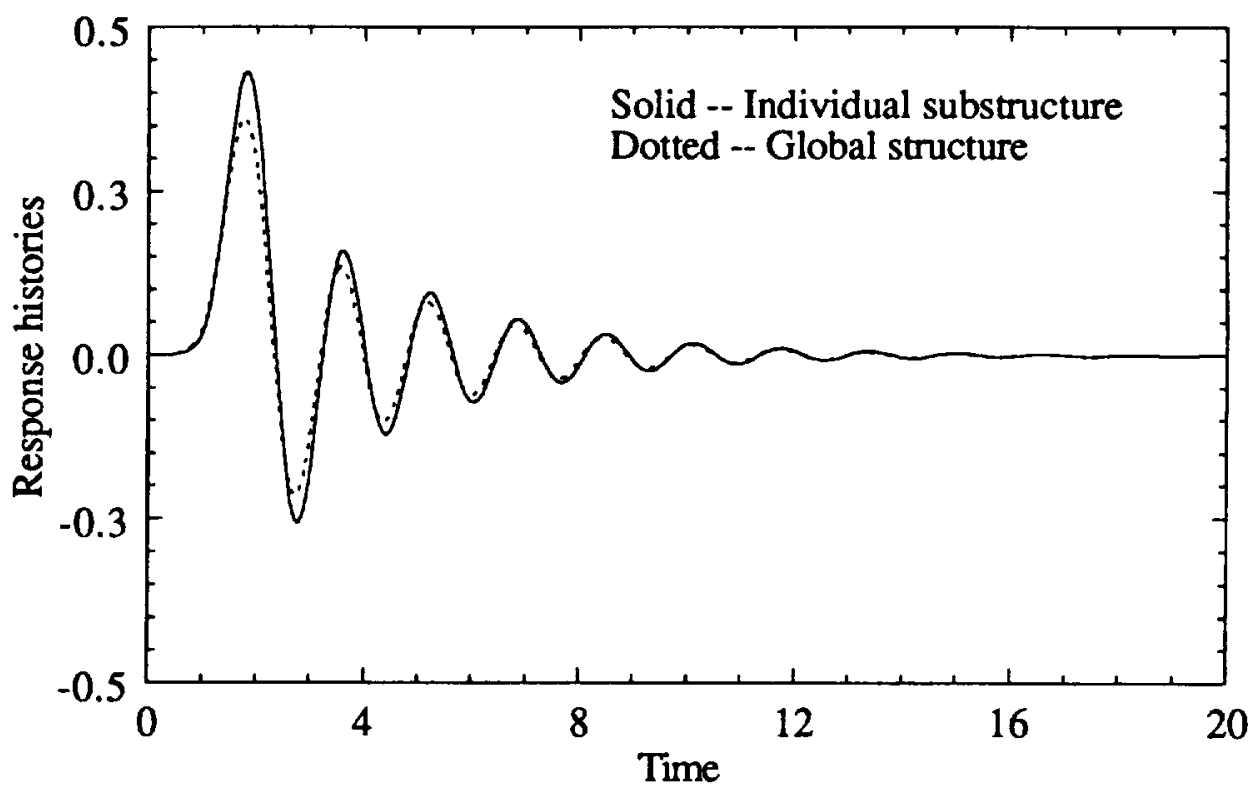

Figure 7: Comparison of closed-loop response histories of DOF 1 (Mass-spring-damper example). 


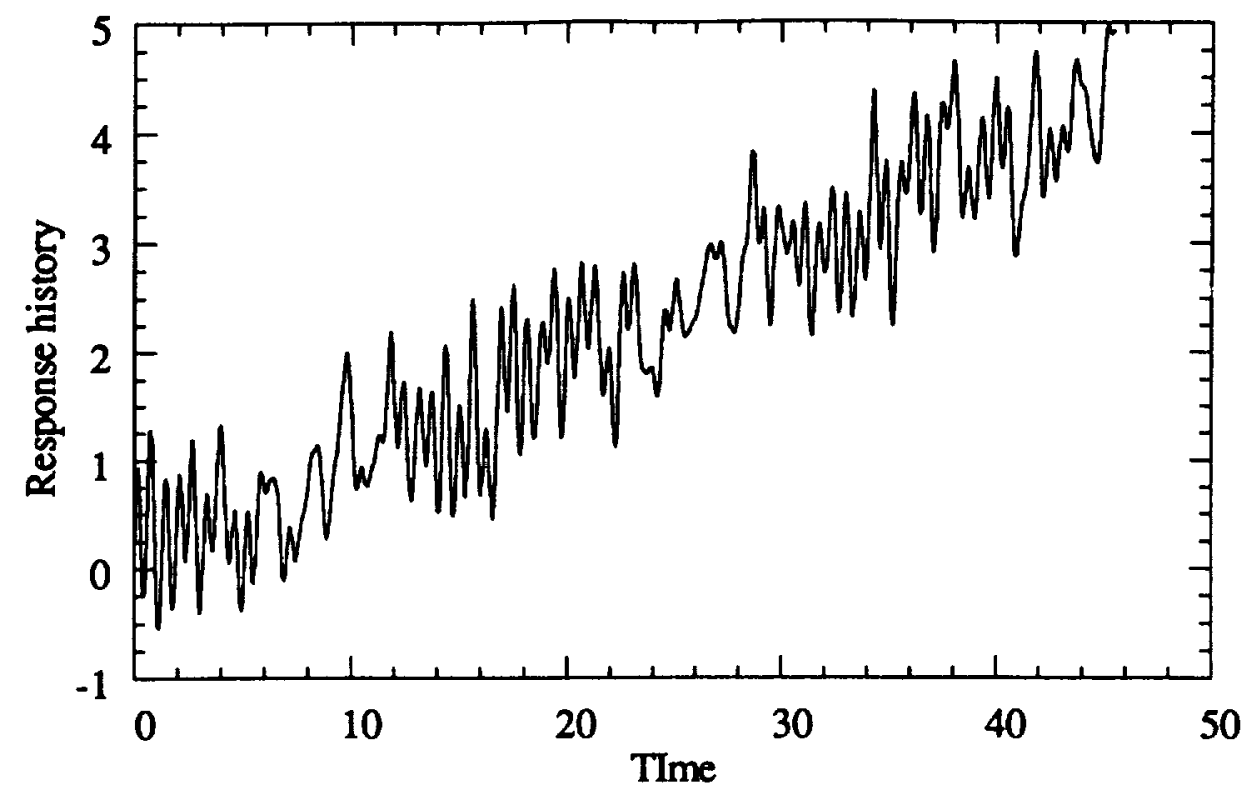

Figure 8: Open-loop response at lower-right corner of $\alpha$ in $x$-direction (Three-truss example).

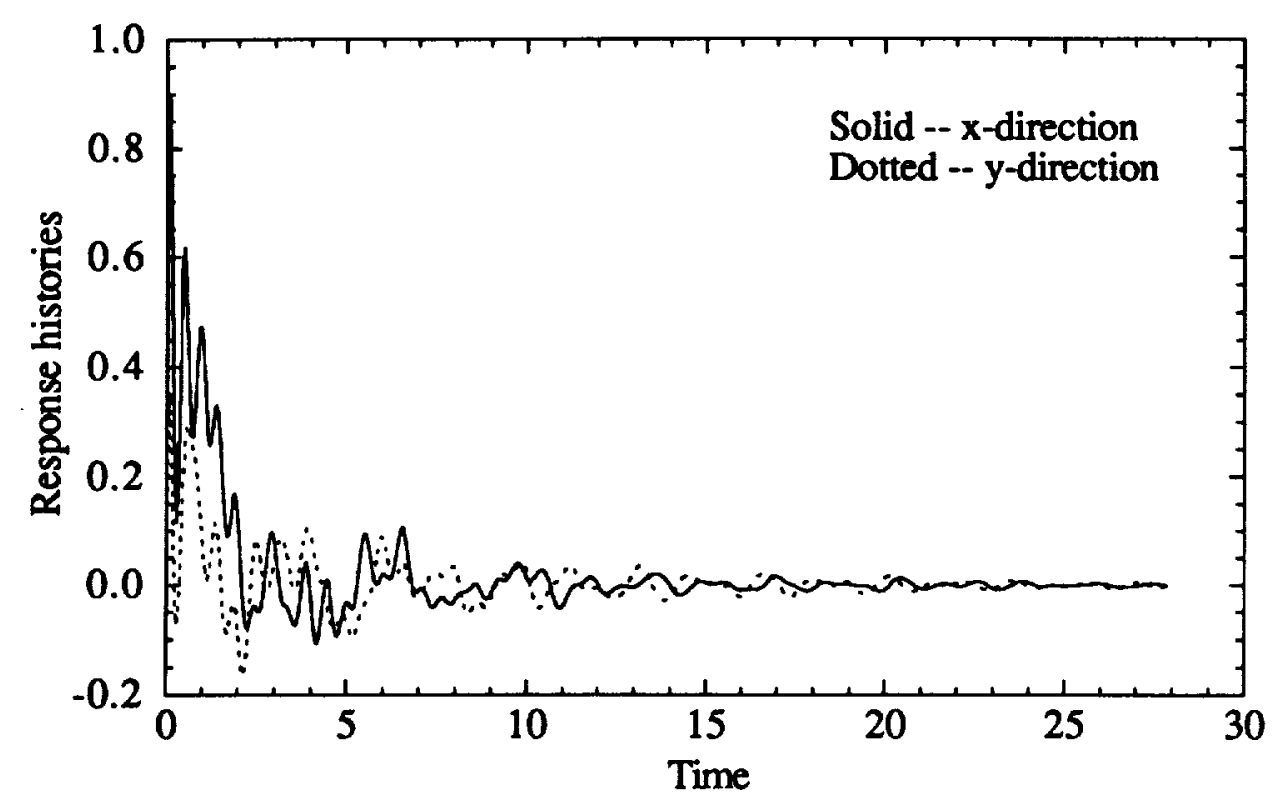

Figure 9: Closed-loop response at lower-right corner of $\alpha$ (Three-truss example). 


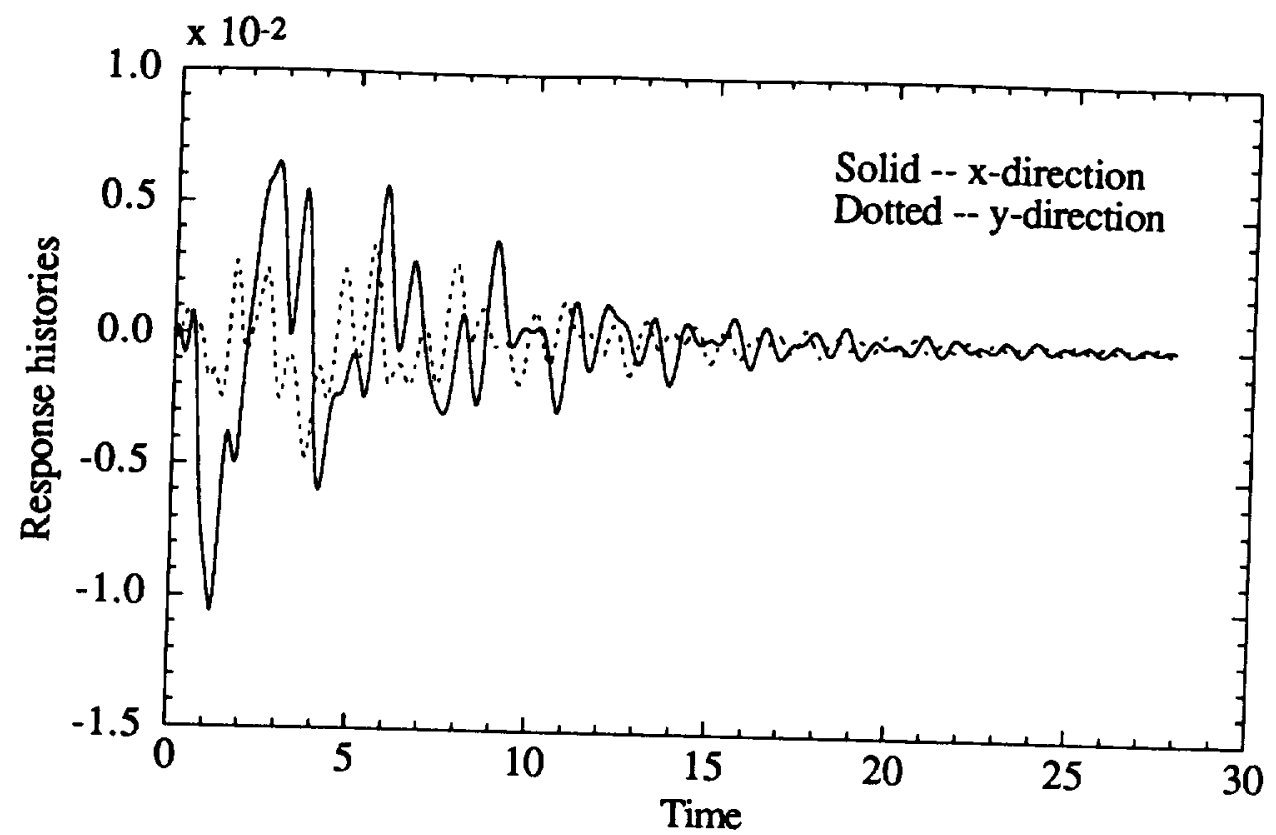

Figure 10: Closed-loop response at lower-left corner of $\beta$ (Three-truss example).

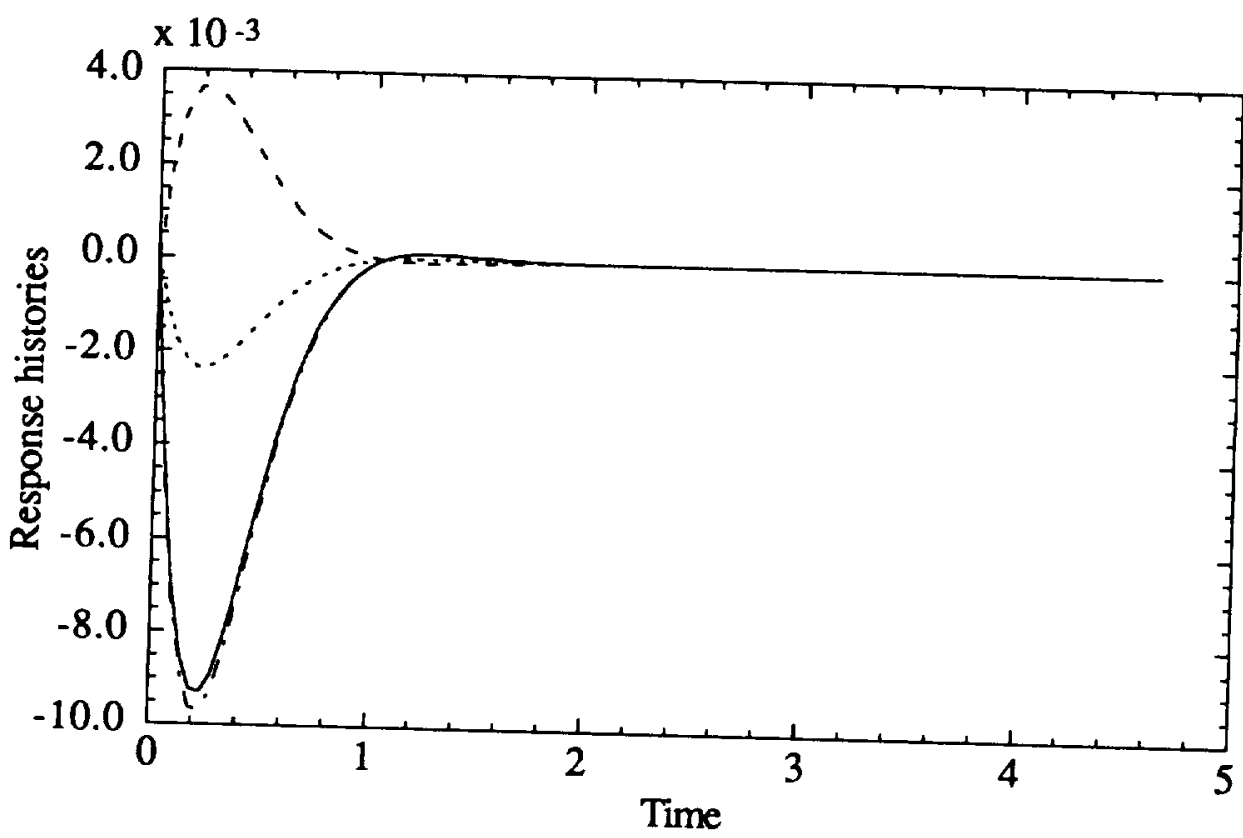

Figure 11: Closed-loop responses of left joint degrees-of-freedom (Three-truss example). 


\section{REPORT DOCUMENTATION PAGE}

Public reponting burden for this collection of intormation is estumated 10 overege I hour per response, including the time for reviewing instructions, searching existing data sourcen

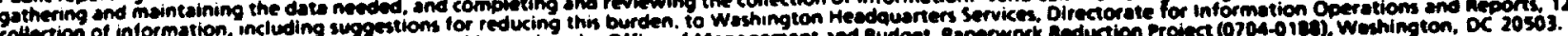

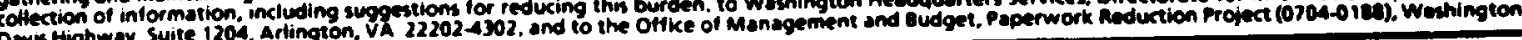

\begin{tabular}{l|l|l|l|l} 
1. AGENCY USE ONLY (leave blank) & 2. REPORT DATE & 3EPORT TYPE AND DATES COVERED
\end{tabular}

October 1992

Technical Memorandum

4. TITLE AND SUBTITLE

Decentralized Control of Large Elexible Structures

By Joint Decoupling

\section{FUNDING NUMBERS}

WU 469-01-11-02

\section{AUTHOR(S)}

Tzu-Jeng Su* and Jer-Nan Juang

\section{PERFORMING ORGANIZATION MAME(S) AND ADDRESS(ES)}

NASA Langley Research Center

Hampton, VA 23681-0001

9. SPONSORING/MONITORING AGENCY NAME(S) AND ADDRESS(ES)

National Aeronautics and Space Administration

washington, DC 20546-0001

8. PERFORMING ORGANIZATION REPORT NUMBER

\section{SUPPLEMENTARY NOTES}

*National Research Council Research Associate, University Affairs Office, NASA Langley Research Center, Hampton, VA 23681-0001

\section{DISTRIBUTION/AVAILABILITY STATEMENT}

126. DISTRIBUTION CODE

Unclassified - Unlimited

Subject Category 39

\section{ABSTRACT (Maximum 200 words)}

This paper presents a decentralized control design method for large complex flexible structures by using the idea of joint decoupling. The derivation is based on a coupled substructure state-space model, which is obtained from enforcing conditions of interface compatibility and equilibrium to the substructure state-space models. It is shown that by restricting the control law to be localized state feedback and by setting the joint actuator input commands to decouple joint degrees-of-freedom from interior degrees-of-freedom, the global structure control design problem can be decomposed into several substructure control design problems. The substructure control gains and substructure observers are designed based on modified substructure state-space models. The controllers produced by the proposed method can operate successfully at the individual substructure level as well as at the global structure level. Therefore, not only control design but also control implementation is decentralized. Stability and performance requirement of the closed-loop system can be achieved by using any existing state feedback control design method. A two-component mass-spring damper system and a three-truss structure are used as examples to demonstrate the proposed method.

\section{SUBJECT TERMS}

Decentralized Control

Decentralized Observer

Substructure Control

17. SECURITY CLASSIFICATION OF REPORT

Unclassified

NSN 7540-01-280-5500
15. MUMBER OF PAGES 30

16. PRLCE CODE

$\mathrm{AO} 3$

\section{SECURITY CIASSIFICATION OF THIS PAgE \\ Unclassified}

19. SECURITY CLASSIFICATION of ABStraCt

Unclassified 